\title{
Strong Well-Posedness of a Diffuse Interface Model for a Viscous, Quasi-Incompressible Two-Phase Flow
}

\author{
Helmut Abels*
}

September 23, 2018

\begin{abstract}
We study a diffuse interface model for the flow of two viscous incompressible Newtonian fluids in a bounded domain. The fluids are assumed to be macroscopically immiscible, but a partial mixing in a small interfacial region is assumed in the model. Moreover, diffusion of both components is taken into account. In contrast to previous works, we study a model for the general case that the fluids have different densities due to Lowengrub and Truskinovski [27]. This leads to an inhomogeneous Navier-Stokes system coupled to a Cahn-Hilliard system, where the density of the mixture depends on the concentration, the velocity field is no longer divergence free, and the pressure enters the equation for the chemical potential. We prove existence of unique strong solutions for the non-stationary system for sufficiently small times.
\end{abstract}

Key words: Two-phase flow, free boundary value problems, diffuse interface model, mixtures of viscous fluids, Cahn-Hilliard equation, inhomogeneous Navier-Stokes equation

AMS-Classification: Primary: 76T99, Secondary: 35Q30, 35Q35, 76D27, 76D03, 76D05, 76D 45

\section{Introduction}

In this article we consider a so-called diffuse interface model for two viscous, incompressible Newtonian fluids of different densities. In the model a partial mixing of the macroscopically immiscible fluids is considered and diffusion effects are taken into account. Such models have been successfully used to describe flows of two or more macroscopically fluids beyond the occurrence of topological singularities of the

\footnotetext{
${ }^{*}$ Fakultät für Mathematik, Universität Regensburg, 93040 Regensburg, Germany, e-mail: helmut.abels@mathematik.uni-regensburg.de
} 
separating interface (e.g. coalescence or formation of drops). We refer to Anderson and McFadden [8] for a review on that topic.

The model which we are considering leads to the system

$$
\begin{array}{rlrl}
\rho \partial_{t} \mathbf{v}+\rho \mathbf{v} \cdot \nabla \mathbf{v}-\operatorname{div} \mathbb{S}(c, \mathbb{D} \mathbf{v})+\nabla p & =-\operatorname{div}(a(c) \nabla c \otimes \nabla c) & & \text { in } Q_{T}, \\
\partial_{t} \rho+\operatorname{div}(\rho \mathbf{v}) & =0 & & \text { in } Q_{T}, \\
\rho \partial_{t} c+\rho \mathbf{v} \cdot \nabla c & =\operatorname{div}(m(c) \nabla \mu) & & \text { in } Q_{T} \\
\rho \mu=-\rho^{-1} \frac{\partial \rho}{\partial c}\left(p+\Phi(c)+\frac{a(c)|\nabla c|^{2}}{2}\right)+\phi(c)-a(c)^{\frac{1}{2}} \operatorname{div}\left(a(c)^{\frac{1}{2}} \nabla c\right)
\end{array}
$$

where $Q_{T}=\Omega \times(0, T)$ and $\Omega \subset \mathbb{R}^{d}, d=2,3$, is a bounded domain with $C^{3}$-boundary. Here $\mathbf{v}$ and $\rho=\hat{\rho}(c)$ are the (mean) velocity and the density of the mixture of the two fluids, $p$ is the pressure, $c$ is the difference of the mass concentrations of the two fluids, and $\mu$ is the chemical potential associated to $c$. Moreover,

$$
\mathbb{S}(c, \mathbb{D} \mathbf{v})=2 \nu(c) \mathbb{D} \mathbf{v}+\eta(c) \operatorname{div} \mathbf{v} \mathbb{I}
$$

where $\mathbb{S}(c, \mathbb{D} \mathbf{v})$ is the stress tensor, $\mathbb{D} \mathbf{v}=\frac{1}{2}\left(\nabla \mathbf{v}+\nabla \mathbf{v}^{T}\right), \nu(c), \eta(c)>0$ are two viscosity coefficients, and $m(c)>0$ is a mobility coefficient. Furthermore, $\Phi(c)$ is the homogeneous free energy density for the mixture and $\phi(c)=\Phi^{\prime}(c)$.

This is a variant of the model proposed by Lowengrub and Truskinovski [27] for an interfacial energy of the form

$$
E_{\text {free }}(c)=\int_{\Omega} \Phi(c) d x+\int_{\Omega} a(c) \frac{|\nabla c|^{2}}{2} d x
$$

where the choice $a(c)=\rho(c)$ was proposed in [27]. A derivation of the latter system can also be found in [1, Chapter II] (in an even more general form). We note that the term $a(c) \nabla c \otimes \nabla c$ comes from an extra contribution to the stress tensor, which models capillary forces in an interfacial region. The model is a generalization of a well-known diffuse interface model in the case of matched densities which corresponds to the case $\hat{\rho}(c) \equiv$ const., cf. e.g. Gurtin et al. [19].

The system is equivalent to

$$
\begin{aligned}
\rho \partial_{t} \mathbf{v}+\rho \mathbf{v} \cdot \nabla \mathbf{v}-\operatorname{div} \mathbb{S}(c, \mathbb{D} \mathbf{v})+\rho \nabla g_{0} & =\rho \mu_{0} \nabla c & & \text { in } Q_{T}, \\
\partial_{t} \rho+\operatorname{div}(\rho \mathbf{v}) & =0 & & \text { in } Q_{T}, \\
\rho \partial_{t} c+\rho \mathbf{v} \cdot \nabla c & =\operatorname{div}\left(m(c) \nabla \mu_{0}\right) & & \text { in } Q_{T}, \\
\rho \mu_{0}+\rho^{2} \bar{p}=\beta \rho^{2} g_{0}-a(c)^{\frac{1}{2}} \operatorname{div}\left(a(c)^{\frac{1}{2}} \nabla c\right) & +\phi(c) & & \text { in } Q_{T},
\end{aligned}
$$

together with

$$
\int_{\Omega} \mu_{0}(t) d x=\int_{\Omega} g_{0}(t) d x=0 \quad \text { for all } t \in(0, T)
$$


where $\mu_{0}$ is the mean-value free part of $\mu$, and $\bar{p}$ is a constant (depending on time), which is related to the mean values of the pressure and the chemical potential. Here $p$ and $g$ are related by

$$
g=\frac{\Phi(c)}{\rho}+\frac{a(c)|\nabla c|^{2}}{2 \rho}+\frac{p}{\rho}-\bar{\mu} c,
$$

and $\mu=\mu_{0}+\bar{\mu}, \bar{\mu}=\frac{1}{|\Omega|} \int_{\Omega} \mu d x, g=g_{0}+\bar{g}, \bar{g}=\frac{1}{|\Omega|} \int_{\Omega} g d x$. Details on this equivalence can be found in [2, Section 3]. Here it is assumed that the fluids mix with zero excess volume, cf. [27]. This implies

$$
\frac{1}{\hat{\rho}(c)}=\frac{1+c}{2 \bar{\rho}_{1}}+\frac{1-c}{2 \bar{\rho}_{2}},
$$

where $\bar{\rho}_{j}$ is the specific density of fluid $j=1,2$. Hence $\hat{\rho}(c)$ is of the form

$$
\hat{\rho}(c)=\frac{1}{\alpha+\beta c}
$$

for $\alpha>0$ and $|\beta|<\alpha$. (More precisely, $\beta=\frac{1}{2 \bar{\rho}_{1}}-\frac{1}{2 \bar{\rho}_{2}}, \alpha=\frac{1}{2 \bar{\rho}_{2}}+\frac{1}{2 \bar{\rho}_{1}}$.)

We close the system by adding the boundary and initial conditions

$$
\begin{aligned}
\left.\mathbf{n} \cdot \mathbf{v}\right|_{\partial \Omega}=(\mathbf{n} \cdot \mathbb{S}(c, \mathbb{D} \mathbf{v}))_{\tau}+\left.\gamma(c) \mathbf{v}_{\tau}\right|_{\partial \Omega} & =0 & & \text { on } S_{T}, \\
\left.\partial_{n} c\right|_{\partial \Omega}=\left.\partial_{n} \mu_{0}\right|_{\partial \Omega} & =0 & & \text { on } S_{T}, \\
\left.(\mathbf{v}, c)\right|_{t=0} & =\left(\mathbf{v}_{0}, c_{0}\right) & & \text { in } \Omega,
\end{aligned}
$$

where $S_{T}=\partial \Omega \times(0, T)$. I.e., we assume that $\mathbf{v}$ satisfies Navier boundary conditions with some friction parameter $\gamma: \mathbb{R} \rightarrow[0, \infty)$ and assume Neumann boundary conditions for $c$ and $\mu$.

In the case of matched densities, i.e., $\hat{\rho} \equiv$ const., $\beta=0$, resp., results on existence of weak solutions and well-posedness were obtained by Starovoitov [33], Boyer [10], Liu and Shen [26], and the author [4. The long time dynamics was studied by Gal and Grasselli [14, 15, 16, Zhao et al. [36] and in [3, 4]. Moreover, in [11] Boyer considered a different diffuse interface model for fluids with non-matched densities. He proved existence of strong solutions, locally in time, and existence of global weak solutions if the densities of the fluids are sufficiently close. In the case of general densities, existence of weak solutions of a slightly modified system was shown in 2, where the case of a free energy of the form

$$
E_{\text {free }}(c)=\int_{\Omega} \Phi(c) d x+\int_{\Omega} a(c) \frac{|\nabla c|^{q}}{q} d x
$$

with $q>d$ is considered. This is the only analytic result for the model (1.1)-(1.4) so far known to the author. To the authors knowledge there are no numerical studies of this model in the case $\beta \neq 0$. A simplified model was used by Lee et al. [24, 25] 
in numerical simulations. Moreover, A. and Feireisl [6] constructed weak solutions globally in time for a corresponding diffuse interface model for compressible fluids.

In the following, we will only consider the case $\rho(c) \not \equiv$ const., i.e., $\beta \neq 0$ and will restrict ourselves for simplicity to the case $a(c)=m(c)=1$. Moreover, we do the following assumption:

Assumption 1.1 Let $\Omega \subset \mathbb{R}^{d}, d=2,3$, be a bounded domain with $C^{3}$-boundary, let $\varepsilon>0, \alpha>0$, and $\beta \neq 0$ such that $|\beta|<\alpha$. Moreover, we assume $\nu, \eta, \gamma \in C^{2}(\mathbb{R})$ such that $\inf _{s \in \mathbb{R}} \nu(s), \inf _{s \in \mathbb{R}} \eta(s)>0$, inf $_{s \in \mathbb{R}} \gamma(s) \geq 0, \Phi \in C^{3}(\mathbb{R})$ and $\hat{\rho} \in C^{3}(\mathbb{R})$ such that $\hat{\rho}(s)=\frac{1}{\alpha+\beta s}$ if $s \in\left[-1-\varepsilon_{0}, 1+\varepsilon_{0}\right]$ for some $\varepsilon_{0}$ and $\hat{\rho}(s)>0$ for all $s \in \mathbb{R}$. Finally, if $\inf _{s \in \mathbb{R}} \gamma(s)=0$, then we assume that $\Omega$ has no axis of symmetry, cf. Appendix for details.

Now our main result on short time existence of strong solutions is:

THEOREM 1.2 Let $\mathbf{v}_{0} \in H_{n}^{1}(\Omega), c_{0} \in H^{2}(\Omega)$ with $\left|c_{0}(x)\right| \leq 1$ almost everywhere and $\left.\partial_{n} c_{0}\right|_{\partial \Omega}=0, d=2,3$, and let Assumption 1.1 hold. Then there is some $T>0$ such that there is a unique solution $\left.\mathbf{v} \in H^{1}\left(0, T ; L_{\sigma}^{2}(\Omega)\right) \cap L^{2}\left(0, T ; H^{2}(\Omega)^{d}\right)\right), c \in$ $H^{2}\left(0, T ; H_{(0)}^{-1}(\Omega)\right) \cap L^{2}\left(0, T ; H^{3}(\Omega)\right)$ solving (1.6) -(1.9),$(1.12)$-(1.14) .

Precise definitions of the function spaces are given in Section 2 below.

The theorem is proved by linearizing the system suitably, proving that the linearized operator defines an isomorphism between certain $L^{2}$-Sobolev spaces, and applying a contraction mapping argument. To apply this general strategy it is essential to reformulate the system (1.6)-(1.9). To this end, we eliminate $\mu_{0}$ and $g_{0}$ first from the system. Then the principal part of the linearized system (around $\left(\mathbf{v}_{0}, c_{0}\right)$ ) is

$$
\begin{aligned}
& \partial_{t} \mathbf{v}-\operatorname{div} \widetilde{\mathbb{S}}\left(c_{0}, \mathbb{D} \mathbf{v}\right)+\frac{\varepsilon}{\beta \alpha} \nabla \operatorname{div}\left(\rho_{0}^{-4} \nabla c^{\prime}\right)=\mathbf{f}_{1} \quad \text { in } Q_{T}, \\
& \partial_{t} c^{\prime}-\beta^{-1} \operatorname{div} \mathbf{v}=f_{2} \quad \text { in } Q_{T},
\end{aligned}
$$

where $c^{\prime} \approx \rho c$ and $\rho_{0}=\hat{\rho}\left(c_{0}\right)$, cf. Section 3 below. One of the essential steps in the proof of the main result is the analysis of this linearized system. To this end we split $\mathbf{v}$ in a divergence free part $\mathbf{w}=P_{\sigma} \mathbf{v}$, a gradient part $\nabla G(\operatorname{div} \mathbf{v})$, which is determined uniquely by $g=\operatorname{div} v$, and a lower order part, cf. Section 4 for details. A crucial observation is that $c^{\prime}$, which is related to div $\mathbf{v}$ via (1.16), solves a kind of damped wave/plate equation. More precisely, $c^{\prime}$ solves an equation of the form

$$
\partial_{t}^{2} c^{\prime}-\Delta\left(a\left(c_{0}\right) \partial_{t} c^{\prime}\right)+\frac{\varepsilon}{\alpha \beta^{2}} \Delta \operatorname{div}\left(\rho_{0}^{-4} \nabla c^{\prime}\right)=f
$$

up to to lower order terms for some $a\left(c_{0}\right)>0$. In order to solve this equation we will apply the abstract result of Chen and Triggiani [12]. - We note that the same kind of linearized system arises in Kotschote [22, where existence of strong solutions locally in time is proved for a compressible Navier-Stokes-Korteweg system. 
Remark 1.3 It is interesting to compare (1.15)-(1.16) to the linearized system of the Model $\mathrm{H}$ for the case of matched densities, i.e, (1.1)-(1.4) in the case when $\beta=0$ and thus $\rho(c) \equiv$ const. Then the pressure $p$ is no longer part of (1.4), $\operatorname{div} \mathbf{v}=0, p$ can no longer be eliminated from the system, and the principal part of the linearized system is

$$
\begin{aligned}
\partial_{t} \mathbf{v}-\operatorname{div} \widetilde{\mathbb{S}}\left(c_{0}, \mathbb{D} \mathbf{v}\right)+\nabla p & =\mathbf{f}_{1} & & \text { in } Q_{T}, \\
\operatorname{div} \mathbf{v} & =0 & & \text { in } Q_{T}, \\
\partial_{t} c+\operatorname{div}\left(m\left(c_{0}\right) \nabla \Delta c\right) & =f_{2} & & \text { in } Q_{T} .
\end{aligned}
$$

Hence the linearized system is very different. In particular, the principal part for $c$ is given by a fourth order diffusion equation with $\sigma(-\operatorname{div}(m(c) \nabla \Delta c)) \subset(-\infty, 0]$ for a suitable realization. While the corresponding operator $A_{1}$ to (1.17) (after reduction to a first order system, cf. Section (4) still generates an analytic semigroup, but the spectral angle $\delta<\frac{\pi}{2}$ can be arbitrarily close to $\frac{\pi}{2}$ in certain situations, cf. Remark 4.3 below. Moreover, note that the Cahn-Hilliard part is decoupled from the NavierStokes part on the level of the principal part of the linearized system in the case $\beta=0$, which is no longer the case if $\beta \neq 0$. - We hope that the insight on the analytic structure of the system (1.6)-(1.9) will help to create stable numerical algorithms, which are not available so far to the best of the author's knowledge.

The structure of the article is as follows: In Section 2 we summarize some notation and preliminary results. The main part of the article consists of Sections 3 and 4 . First, in Section 3 the system is linearized and the contraction mapping principle is applied on the basis of the well-posedness result Theorem 3.1 for the linearized system. Afterwards, in Section 4 this result is proved.

\section{Preliminaries}

Notation: Let us fix some notation first. For $a, b \in \mathbb{R}^{d}$ let $a \otimes b \in \mathbb{R}^{d \times d}$ be defined by $(a \otimes b)_{i, j}=a_{i} b_{j}$. Moreover, for $A, B \in \mathbb{R}^{d \times d}$ let $A: B=\operatorname{tr}\left(A^{T} B\right)=\sum_{i, j=1}^{d} a_{i, j} b_{i, j}$. In the following $\mathbf{n}$ will denote the exterior normal at the boundary of a sufficiently smooth domain $\Omega \subset \mathbb{R}^{d}$. Furthermore, $\mathbf{f}_{n}:=\mathbf{n} \cdot \mathbf{f}$ and $\mathbf{f}_{\tau}:=(I-\mathbf{n} \otimes \mathbf{n}) f=$ $\mathbf{f}-\mathbf{f}_{n} \mathbf{n}$ denote the normal and tangential component of a vector field $\mathbf{f}: \partial \Omega \rightarrow \mathbb{R}^{d}$, respectively. Furthermore $\partial_{n}:=\mathbf{n} \cdot \nabla, \nabla_{\tau}:=(I-\mathbf{n} \otimes \mathbf{n}) \nabla$, and $\partial_{\tau_{j}}:=e_{j} \cdot(I-\mathbf{n} \otimes \mathbf{n}) \nabla$, $j=1, \ldots, d$, where $e_{j}$ denotes the $j$-th canonical unit vector in $\mathbb{R}^{d}$. If $\mathbf{v} \in C^{1}(\Omega)^{d}$, then $\nabla \mathbf{v}=D \mathbf{v}=\left(\partial_{x_{j}} v_{i}\right)_{i, j=1}^{d}$ denotes its Jacobian. Moreover, if $A=\left(a_{i j}\right)_{i, j=1}^{d}: \Omega \rightarrow$ $\mathbb{R}^{d \times d}$ is differentiable, then $\operatorname{div} A(x):=\left(\sum_{j=1}^{d} \partial_{x_{j}} a_{i j}(x)\right)_{i=1}^{d}$ for all $x \in \Omega$.

If $X$ is a Banach space and $X^{\prime}$ is its dual, then

$$
\langle f, g\rangle \equiv\langle f, g\rangle_{X^{\prime}, X}=f(g), \quad f \in X^{\prime}, g \in X,
$$

denotes the duality product. The inner product on a Hilbert space $H$ is denoted by $(., .)_{H}$. Moreover, we use the abbreviation $(., .)_{M}=(.,)_{L^{2}(M)}$. 
Function spaces: If $M \subseteq \mathbb{R}^{d}$ is measurable, $L^{q}(M), 1 \leq q \leq \infty$ denotes the usual Lebesgue-space and $\|\cdot\|_{q}$ its norm. Moreover, $L^{q}(M ; X)$ denotes its vector-valued variant of strongly measurable $q$-integrable functions/essentially bounded functions, where $X$ is a Banach space. If $M=(a, b)$, we write for simplicity $L^{q}(a, b ; X)$ and $L^{q}(a, b)$.

Let $\Omega \subset \mathbb{R}^{d}$ be a domain. Then $W_{q}^{m}(\Omega), m \in \mathbb{N}_{0}, 1 \leq q \leq \infty$, denotes the usual $L^{q}$-Sobolev space, $W_{q, 0}^{m}(\Omega)$ the closure of $C_{0}^{\infty}(\Omega)$ in $W_{q}^{m}(\Omega), W_{q}^{-m}(\Omega)=\left(W_{q^{\prime}, 0}^{m}(\Omega)\right)^{\prime}$, and $W_{q, 0}^{-m}(\Omega)=\left(W_{q^{\prime}}^{m}(\Omega)\right)^{\prime}$. The $L^{2}$-Bessel potential spaces are denoted by $H^{s}(\Omega)$, $s \in \mathbb{R}$, which are defined by restriction of distributions in $H^{s}\left(\mathbb{R}^{d}\right)$ to $\Omega$, cf. Triebel [35, Section 4.2.1]. Moreover, $H^{s}(M)$ denotes the corresponding space on a sufficiently smooth compact manifold $M$. We note that, if $\Omega \subset \mathbb{R}^{d}$ is a bounded domain with $C^{0,1}$-boundary, then there is an extension operator $E_{\Omega}$ which is a bounded linear operator $E_{\Omega}: W_{p}^{m}(\Omega) \rightarrow W_{p}^{m}\left(\mathbb{R}^{d}\right), 1 \leq p \leq \infty$ for all $m \in \mathbb{N}$ and $\left.E_{\Omega} f\right|_{\Omega}=f$ for all $f \in W_{p}^{m}(\Omega)$, cf. Stein [34, Chapter VI, Section 3.2]. This extension operator extends to $E_{\Omega}: H^{s}(\Omega) \rightarrow H^{s}\left(\mathbb{R}^{n}\right)$, which shows that $H^{s}(\Omega)$ is a retract of $H^{s}(\Omega)$. Therefore all results on interpolation spaces of $H^{s}\left(\mathbb{R}^{n}\right)$ carry over to $H^{s}(\Omega)$. We refer to Bergh and Löfström [9] for basic results in interpolation theory. In the following $(., .)_{[\theta]}$ and $(., .)_{\theta, q}$ will denote the complex and real interpolation function, respectively. In particular, we note that

$$
\left(H^{s_{0}}(\Omega), H^{s_{1}}(\Omega)\right)_{[\theta]}=\left(H^{s_{0}}(\Omega), H^{s_{1}}(\Omega)\right)_{\theta, 2}=H^{s}(\Omega)
$$

for all $\theta \in(0,1)$ where $s=(1-\theta) s_{0}+\theta s_{1}, s_{0}, s_{1} \in \mathbb{R}$.

Moreover, we define

$$
H_{n}^{1}(\Omega)=\left\{\mathbf{u} \in H^{1}(\Omega)^{d}:\left.\mathbf{n} \cdot \mathbf{u}\right|_{\partial \Omega}=0\right\} .
$$

The usual Besov spaces on a domain or a sufficiently smooth manifold are denoted by $B_{p, q}^{s}(\Omega), B_{p, q}^{s}(M)$, resp., where $s \in \mathbb{R}, 1 \leq p, q \leq \infty$. For the convenience of the reader we recall that $B_{2,2}^{s}\left(\mathbb{R}^{d}\right)=H^{s}\left(\mathbb{R}^{d}\right)$ for all $s \in \mathbb{R}$ and

$$
B_{p, \infty}^{s+\varepsilon}\left(\mathbb{R}^{d}\right) \hookrightarrow B_{p, q_{1}}^{s}\left(\mathbb{R}^{d}\right) \hookrightarrow B_{p, q_{2}}^{s}\left(\mathbb{R}^{d}\right) \quad \text { for all } 1 \leq q_{1} \leq q_{2} \leq \infty .
$$

Moreover, we have the Sobolev type embeddings

$$
\begin{aligned}
B_{p_{1}, q}^{s_{1}}\left(\mathbb{R}^{n}\right) & \hookrightarrow B_{p_{0}, q}^{s_{0}}\left(\mathbb{R}^{n}\right) \quad \text { if } s_{1} \geq s_{0} \text { and } s_{1}-\frac{n}{p_{1}} \geq s_{0}-\frac{n}{p_{0}}, \\
B_{p, 1}^{d / p}\left(\mathbb{R}^{n}\right) & \hookrightarrow C_{b}^{0}\left(\mathbb{R}^{n}\right)
\end{aligned}
$$

for any $1 \leq p, q \leq \infty$. Finally, due to Hanouzet [20, Théorème 3] we have the useful product estimate

$$
\|f g\|_{H^{1}\left(\mathbb{R}^{d}\right)} \leq C_{p}\|f\|_{B_{p, 1}^{d / p}\left(\mathbb{R}^{d}\right)}\|g\|_{H^{1}\left(\mathbb{R}^{d}\right)}
$$

for all $f \in B_{p, 1}^{\frac{d}{p}}\left(\mathbb{R}^{d}\right), g \in H^{1}\left(\mathbb{R}^{d}\right)$ provided that $2 \leq p \leq \infty$, see also [21, Theorem 6.6]. All these results carry over to sufficiently smooth domains and $d$-dimensional manifolds. 
Let $I=[0, T]$ with $0<T<\infty$ or let $I=[0, \infty)$ and let $X$ be a Banach space. Then $B U C(I ; X)$ is the Banach space of all bounded and uniformly continuous $f: I \rightarrow X$ equipped with the supremum norm. The space of all (uniformly) Hölder continuous functions $f: I \rightarrow X$ of degree $s \in(0,1)$ is denoted by $C^{s}([0, T] ; X)$ normed in the standard way. Furthermore, we have the useful embedding

$$
B U C\left([0, T] ; X_{1}\right) \cap C^{s}\left([0, T] ; X_{0}\right) \hookrightarrow C^{s(1-\theta)}([0, T] ; X),
$$

where $0<s, \theta<1$ provided that $\|f\|_{X} \leq C\|f\|_{X_{0}}^{1-\theta}\|f\|_{X_{1}}^{\theta}$ for all $f \in X_{0} \cap X_{1}$.

Finally, $f \in W_{p}^{k}(0, T ; X), 1 \leq p<\infty, k \in \mathbb{N}_{0}$, if and only if $f, \ldots, \frac{d^{k} f}{d t^{k}} \in$ $L^{p}(0, T ; X)$, where $\frac{d^{k} f}{d t^{k}}$ denotes the $k$-th $X$-valued distributional derivative of $f$. Furthermore, we set $H^{1}(0, T ; X)=W_{2}^{1}(0, T ; X)$ and for $s \in(0,1)$ we define $H^{s}(0, T ; X)=$ $B_{2,2}^{s}(0, T ; X)$, where $f \in B_{2,2}^{s}(0, T ; X)$ if and only if $f \in L^{2}(0, T ; X)$ and

$$
\|f\|_{B_{2,2}^{s}(0, T ; X)}^{2}=\|f\|_{L^{2}(0, T ; X)}^{2}+\int_{0}^{T} \int_{0}^{T} \frac{\|f(t)-f(\tau)\|_{X}^{2}}{|t-\tau|^{2 s+1}} d t d \tau<\infty .
$$

In the following we will use that

$$
\begin{aligned}
& \int_{0}^{T} \int_{0}^{T} \frac{\|f(t)-f(\tau)\|_{X}^{2}}{|t-\tau|^{2 s+1}} d t d \tau \\
& \quad \leq \int_{0}^{T} \int_{0}^{T}|t-\tau|^{2\left(s^{\prime}-s\right)-1} d t d \tau\|f\|_{C^{s^{\prime}([0, T] ; X)}} \leq C_{s^{\prime}, s} T^{2\left(s^{\prime}-s\right)+1}\|f\|_{C^{s^{\prime}([0, T] ; X)}}
\end{aligned}
$$

for all $0<s<s^{\prime} \leq 1$, which implies

$$
\|f\|_{H^{s}(0, T ; X)} \leq C_{s, s^{\prime}} T^{\frac{1}{2}}\|f\|_{C^{s^{\prime}([0, T] ; X)}} \quad \text { for all } f \in C^{s^{\prime}}([0, T] ; X)
$$

provided that $0<s<s^{\prime} \leq 1$. Finally, we set for $s \in(0,1)$

$$
H^{\frac{s}{2}, s}\left(S_{T}\right)=L^{2}\left(0, T ; H^{s}(\partial \Omega)\right) \cap H^{\frac{s}{2}}\left(0, T ; L^{2}(\partial \Omega)\right),
$$

where $S_{T}=\partial \Omega \times(0, T)$ and $\Omega$ is a bounded domain with $C^{1}$-boundary.

Now let $X_{0}, X_{1}$ be Banach spaces such that $X_{1} \hookrightarrow X_{0}$ densely. Then

$$
W_{p}^{1}\left(I ; X_{0}\right) \cap L^{p}\left(I ; X_{1}\right) \hookrightarrow B U C\left(I ;\left(X_{0}, X_{1}\right)_{1-\frac{1}{p}, p}\right), \quad 1 \leq p<\infty,
$$

continuously for $I=[0, T], 0<T<\infty$, and $I=[0, \infty)$, cf. Amann [7, Chapter III, Theorem 4.10.2].

In order to solve the linearized system in the following, we will use the following abstract result:

THEOREM 2.1 Let $A: \mathcal{D}(A) \subset H \rightarrow H$ be a generator of a bounded analytic semigroup on a Hilbert space $H$ and let $1<q<\infty$. Then for every $f \in L^{q}(0, \infty ; H)$ and 
$u_{0} \in(H, \mathcal{D}(A))_{1-\frac{1}{q}, q}$ there is a unique $u:[0, \infty) \rightarrow H$ such that $\frac{d u}{d t}, A u \in L^{q}(0, \infty ; H)$ solving

$$
\begin{aligned}
\frac{d u}{d t}(t)+A u(t) & =f(t) \quad \text { for all } t>0 \\
u(0) & =u_{0} .
\end{aligned}
$$

Moreover, there is a constant $C_{q}>0$ independent of $f$ and $u_{0}$ such that

$$
\left\|\frac{d u}{d t}\right\|_{L^{q}(0, \infty ; H)}+\|A u\|_{L^{q}(0, \infty ; H)} \leq C_{q}\left(\|f\|_{L^{q}(0, \infty ; H)}+\left\|u_{0}\right\|_{(H, \mathcal{D}(A))_{1-\frac{1}{q}, q}}\right) .
$$

Proof: In the case $u_{0}=0$ the statement is the main result of [13]. The general case can be easily reduced to the case $u_{0}=0$ by subtracting a suitable extension. The existence of such an extension follows e.g. from [7, Chapter III, Theorem 4.10.2].

Weak Neumann Laplace equation: In the following we assume that $\Omega \subset \mathbb{R}^{d}$ is a bounded domain with $C^{0,1}$-boundary. Given $f \in L^{1}(\Omega)$, we denote by $m(f)=$ $\frac{1}{|\Omega|} \int_{\Omega} f(x) d x$ its mean value. Moreover, for $m \in \mathbb{R}$ we set

$$
L_{(m)}^{q}(\Omega):=\left\{f \in L^{q}(\Omega): m(f)=m\right\}, \quad 1 \leq q \leq \infty
$$

Then

$$
P_{0} f:=f-m(f)=f-\frac{1}{|\Omega|} \int_{\Omega} f(x) d x
$$

is the orthogonal projection onto $L_{(0)}^{2}(\Omega)$. Furthermore, we define

$$
H_{(0)}^{1} \equiv H_{(0)}^{1}(\Omega)=H^{1}(\Omega) \cap L_{(0)}^{2}(\Omega), \quad(c, d)_{H_{(0)}^{1}(\Omega)}:=(\nabla c, \nabla d)_{L^{2}(\Omega)} .
$$

Then $H_{(0)}^{1}(\Omega)$ is a Hilbert space due to Poincaré's inequality

$$
\|f-m(f)\|_{L^{p}(\Omega)} \leq C_{p}\|\nabla f\|_{L^{p}(\Omega)},
$$

where $1 \leq p<\infty$. Moreover, let $H_{(0)}^{-1} \equiv H_{(0)}^{-1}(\Omega)=H_{(0)}^{1}(\Omega)^{\prime}$. Then the weak Neumann-Laplace operator $\Delta_{N}: H_{(0)}^{1}(\Omega) \rightarrow H_{(0)}^{-1}(\Omega)$ is defined by

$$
-\left\langle\Delta_{N} u, \varphi\right\rangle_{H_{(0)}^{1}, H_{(0)}^{-1}}=(\nabla u, \nabla \varphi) \quad \text { for all } \varphi \in H_{(0)}^{1}(\Omega)
$$

By the Lemma of Lax-Milgram, for every $f \in H_{(0)}^{-1}(\Omega)$ there is a unique $u \in H_{(0)}^{1}(\Omega)$ such that $-\Delta_{N} u=f$. More precisely, $-\Delta_{N}$ coincides with the Riesz isomorphism $\mathcal{R}: H_{(0)}^{1}(\Omega) \rightarrow H_{(0)}^{-1}(\Omega)$ given by

$$
\langle\mathcal{R} c, d\rangle_{H_{(0)}^{-1}, H_{(0)}^{1}}=(c, d)_{H_{(0)}^{1}}=(\nabla c, \nabla d)_{L^{2}}, \quad c, d \in H_{(0)}^{1}(\Omega)
$$


We equip $H_{(0)}^{-1}(\Omega)$ with the inner product

$$
(f, g)_{H_{(0)}^{-1}}=\left(\nabla \Delta_{N}^{-1} f, \nabla \Delta_{N}^{-1} g\right)_{L^{2}}=\left(\Delta_{N}^{-1} f, \Delta_{N}^{-1} g\right)_{H_{(0)}^{1}} .
$$

In particular this implies the useful identity

$$
\left(\left(-\Delta_{N}\right) f, g\right)_{H_{(0)}^{-1}}=(f, g)_{L^{2}} \quad \text { for all } f \in H_{(0)}^{1}(\Omega), g \in L_{(0)}^{2}(\Omega) .
$$

Moreover, we embed $H_{(0)}^{1}(\Omega)$ and $L_{(0)}^{2}(\Omega)$ into $H_{(0)}^{-1}(\Omega)$ in the standard way by defining

$$
\langle c, \varphi\rangle_{H_{(0)}^{-1}, H_{(0)}^{1}}=\int_{\Omega} c(x) \varphi(x) d x \quad \text { for all } \varphi \in H_{(0)}^{1}(\Omega)
$$

for $c \in L_{(0)}^{2}(\Omega)$. This implies the useful interpolation inequality

$$
\|f\|_{L^{2}}^{2}=-\left(\nabla \Delta_{N}^{-1} f, \nabla f\right)_{L^{2}} \leq\|f\|_{H_{(0)}^{-1}}\|f\|_{H_{(0)}^{1}} \quad \text { for all } f \in H_{(0)}^{1}(\Omega) .
$$

Furthermore, if $\Delta_{N} u=f$ for some $f \in H_{(0)}^{-1}(\Omega)$, then

$$
\|u\|_{H_{(0)}^{1}(\Omega)} \leq\|f\|_{H_{(0)}^{-1}(\Omega)} .
$$

We note that, if $u \in H_{(0)}^{1}(\Omega)$ solves $\Delta_{N} u=f$ for some $f \in L_{(0)}^{q}(\Omega), 1<q<\infty$, and $\partial \Omega$ is of class $C^{2}$, then it follows from standard elliptic theory that $u \in W_{q}^{2}(\Omega)$, $\Delta u=f$ a.e. in $\Omega$, and $\left.\partial_{n} u\right|_{\partial \Omega}=0$ in the sense of traces. If additionally $f \in W_{q}^{1}(\Omega)$ and $\partial \Omega \in C^{3}$, then $u \in W_{q}^{3}(\Omega)$. Moreover,

$$
\|u\|_{W_{q}^{k+2}(\Omega)} \leq C_{q}\|f\|_{W_{q}^{k}(\Omega)} \quad \text { for all } f \in W_{q}^{k}(\Omega) \cap L_{(0)}^{q}(\Omega), k=0,1,
$$

with a constant $C_{q}$ depending only on $1<q<\infty, d, k$, and $\Omega$.

Finally, we define $\operatorname{div}_{n}: L^{2}(\Omega) \rightarrow H_{(0)}^{-1}(\Omega)$ by

$$
\left\langle\operatorname{div}_{n} f, \varphi\right\rangle_{H_{(0)}^{-1}(\Omega), H_{(0)}^{1}(\Omega)}=-(f, \nabla \varphi)_{L^{2}(\Omega)} \quad \text { for all } \varphi \in H_{(0)}^{1}(\Omega) .
$$

Note that $\Delta_{N} u=\operatorname{div}_{n} \nabla u$ for all $u \in H_{(0)}^{1}(\Omega)$.

Helmholtz decomposition: Recall that we have the orthogonal decomposition

$$
\begin{aligned}
L^{2}(\Omega)^{d} & =L_{\sigma}^{2}(\Omega) \oplus G_{2}(\Omega) \\
G_{2}(\Omega) & =\left\{\nabla p \in L^{2}(\Omega): p \in H_{(0)}^{1}(\Omega)\right\} .
\end{aligned}
$$

Here $L_{\sigma}^{2}(\Omega)$ is the closure of $\left\{\mathbf{u} \in C_{0}^{\infty}(\Omega)^{d}: \operatorname{div} \mathbf{u}=0\right\}$ in $L^{2}(\Omega)^{d}$. The Helmholtz projection $P_{\sigma}$ is the orthogonal projection onto $L_{\sigma}^{2}(\Omega)$. We note that $P_{\sigma} f=f-\nabla p$, where $p \in H_{(0)}^{1}(\Omega)$ is the solution of the weak Neumann problem

$$
(\nabla p, \nabla \varphi)_{\Omega}=(f, \nabla \varphi) \text { for all } \varphi \in C_{(0)}^{\infty}(\bar{\Omega}) .
$$

We refer to Simader and Sohr [31] and Sohr [32, Chapter II, Section 2.5] for details.

We conclude this section with two technical results related to the Navier boundary condition (1.12), which will be needed in Section 4 . 
Lemma 2.2 Let $\Omega \subset \mathbb{R}^{d}$ be a bounded domain with $C^{k}$-boundary, $k \geq 2$. Then there is a first order tangential differential operator $A=\sum_{j=1}^{d} a_{j}(x) \partial_{\tau_{j}}, a_{j} \in C^{k-1}(\partial \Omega)$, such that

$$
\left.\left(\mathbf{n} \cdot \nabla^{2} u\right)_{\tau}\right|_{\partial \Omega}=\nabla_{\tau} \gamma_{1} u+A \gamma_{0} u \quad \text { for all } u \in H^{2}(\Omega),
$$

where $\gamma_{j} u=\left.\partial_{n}^{j} u\right|_{\partial \Omega}$.

Proof: Since $\nabla_{\tau}=(I-\mathbf{n} \otimes \mathbf{n}) \nabla$, we obtain

$$
\left.\left(\mathbf{n} \cdot \nabla^{2} u\right)_{\tau}\right|_{\partial \Omega}=\left.(I-\mathbf{n} \otimes \mathbf{n})\left(\mathbf{n} \cdot \nabla^{2} u\right)\right|_{\partial \Omega}=(I-\mathbf{n} \otimes \mathbf{n}) \nabla\left(\partial_{n} u\right)-\sum_{j=1}^{d}\left(\partial_{\tau_{j}} \mathbf{n}\right) \cdot \nabla u
$$

for all $u \in H^{2}(\Omega)$. Since $\partial_{\tau_{j}} \mathbf{n} \cdot \mathbf{n}=\frac{1}{2} \partial_{\tau_{j}}|\mathbf{n}|^{2}=0, \partial_{\tau_{j}} \mathbf{n} \in C^{k-1}(\partial \Omega)$ is tangential. Therefore (2.11) is valid.

Lemma 2.3 Let $\Omega \subset \mathbb{R}^{d}$ be a bounded domain with $C^{2}$-boundary, $0<T \leq \infty$, $\nu \in C^{1}(\mathbb{R})$ with $\inf _{s \in \mathbb{R}} \nu(s)>0$, and $c_{0} \in H^{2}(\Omega), d=2,3$.

1. There is a bounded linear operator $E: H^{\frac{1}{2}}(\partial \Omega)^{d} \rightarrow H^{2}(\Omega)^{d}$ such that

$$
\left.(\mathbf{n} \cdot 2 \mathbb{D} E \mathbf{a})_{\tau}\right|_{\partial \Omega}=\mathbf{a}_{\tau},\left.\quad E \mathbf{a}\right|_{\partial \Omega}=0, \quad \operatorname{div} E \mathbf{a}=0
$$

for all $\mathbf{a} \in H^{\frac{1}{2}}(\partial \Omega)^{d}$. Moreover, there is a constant $C>0$ such that $\|E a\|_{H^{1}(\Omega)} \leq$ $C\|a\|_{H^{-\frac{1}{2}(\partial \Omega)}}$ for all $a \in H^{\frac{1}{2}}(\partial \Omega)^{d}$.

2. There is a bounded linear operator

$$
E_{T}: H^{\frac{1}{4}, \frac{1}{2}}\left(S_{T}\right)^{d} \rightarrow L^{2}\left(0, T ; H^{2}(\Omega)\right)^{d} \cap H^{1}\left(0, T ; L_{\sigma}^{2}(\Omega)\right)
$$

such that

$$
\left.\left(\mathbf{n} \cdot 2 \nu\left(c_{0}\right) \mathbb{D} E_{T} \mathbf{a}\right)_{\tau}\right|_{\partial \Omega}=\mathbf{a}_{\tau},\left.\quad E_{T} \mathbf{a}\right|_{\partial \Omega}=0, \quad \operatorname{div} E_{T} \mathbf{a}=0,\left.\quad E_{T} \mathbf{a}\right|_{t=0}=0
$$

for all $\mathbf{a} \in H^{\frac{1}{4}, \frac{1}{2}}\left(S_{T}\right)^{d}$. Moreover, the operator norm of $E_{T}$ can be estimated independently of $0<T \leq \infty$.

Proof: To prove the first part let $\tilde{\mathbf{A}}=\tilde{E} \mathbf{a} \in H^{2}(\Omega)^{d}$ such that $\left.\tilde{\mathbf{A}}\right|_{\partial \Omega}=0,\left.\partial_{n} \tilde{\mathbf{A}}\right|_{\partial \Omega}=$ $\mathbf{a}_{\tau}$ and $\|\tilde{\mathbf{A}}\|_{H^{2}(\Omega)} \leq C\|\mathbf{a}\|_{H^{\frac{1}{2}(\partial \Omega)}},\|\tilde{\mathbf{A}}\|_{H^{1}(\Omega)} \leq C\|\mathbf{a}\|_{H^{-\frac{1}{2}(\partial \Omega)}}$ for all $\mathbf{a} \in H^{\frac{1}{2}}(\partial \Omega)^{d}$. If $\Omega=\mathbb{R}^{n-1} \times(0, \infty)$, the existence of such an extension operator $\tilde{E}$ follows e.g. from McLean [28, Lemma 3.36]. From this the result for a general bounded $C^{2}$-domain follows by standard localization techniques.

Then we have

$$
\begin{aligned}
\left.(\mathbf{n} \cdot 2 \mathbb{D} \tilde{\mathbf{A}})_{\tau}\right|_{\partial \Omega} & =\left.\left(\nabla_{\tau} \tilde{\mathbf{A}}_{n}+\partial_{n} \tilde{\mathbf{A}}_{\tau}\right)\right|_{\partial \Omega}=0+\mathbf{a}_{\tau} \\
\left.\operatorname{div} \tilde{\mathbf{A}}\right|_{\partial \Omega} & =\left.\left(\operatorname{div}_{\tau} \tilde{\mathbf{A}}+\partial_{n} \tilde{\mathbf{A}}_{n}\right)\right|_{\partial \Omega}=0
\end{aligned}
$$


Since $\left.\operatorname{div} \tilde{\mathbf{A}}\right|_{\partial \Omega}=0, \operatorname{div} \tilde{\mathbf{A}} \in H_{0}^{1}(\Omega) \cap L_{(0)}^{2}(\Omega)$ and we can apply the Bogovski-Operator $B$, cf. e.g. [17], to $\operatorname{div} \tilde{\mathbf{A}}$. Hence we obtain $B(\operatorname{div} \tilde{\mathbf{A}}) \in H_{0}^{2}(\Omega), \operatorname{div} B(\operatorname{div} \tilde{\mathbf{A}})=\operatorname{div} \tilde{\mathbf{A}}$, and

$$
\begin{aligned}
\|B(\operatorname{div} \tilde{\mathbf{A}})\|_{H^{2}(\Omega)} & \leq C\|\tilde{\mathbf{A}}\|_{H^{2}(\Omega)} \leq C^{\prime}\|\mathbf{a}\|_{H^{\frac{1}{2}(\partial \Omega)}}, \\
\|B(\operatorname{div} \tilde{\mathbf{A}})\|_{H^{1}(\Omega)} & \leq C\|\tilde{\mathbf{A}}\|_{H^{1}(\Omega)} \leq C^{\prime}\|\mathbf{a}\|_{H^{-\frac{1}{2}}(\partial \Omega)}
\end{aligned}
$$

for all $\mathbf{a} \in H^{\frac{1}{2}}(\partial \Omega)$. Therefore $A:=\tilde{A}-B(\operatorname{div} \tilde{A})$ has the properties stated above.

Finally, because of [5, Lemma 2.4], for every $\mathbf{a} \in H^{\frac{1}{4}}, \frac{1}{2}\left(S_{T}\right)^{d}$ there is some $\mathbf{A} \in$ $L^{2}\left(0, T ; H^{1}(\Omega)\right)^{d} \cap H^{1}\left(0, T ; L^{2}(\Omega)\right)^{d}$ such that $\left.\left(\mathbf{n} \cdot \nu\left(c_{0}\right) \mathbb{D} \mathbf{A}\right)_{\tau}\right|_{\partial \Omega}=\mathbf{a}_{\tau},\left.\mathbf{A}\right|_{t=0}=0$, $\left.\operatorname{div} \mathbf{A}\right|_{\partial \Omega}=0,\left.\mathbf{A}\right|_{\partial \Omega}=0$. Moreover, the extension can be chosen such that

$$
\|\mathbf{A}\|_{L^{2}\left(0, T ; H^{2}\right)}+\|\mathbf{A}\|_{H^{1}\left(0, T ; L^{2}\right)} \leq C\|\mathbf{a}\|_{H^{\frac{1}{4}, \frac{1}{2}}\left(S_{T}\right)}
$$

with $C$ independent of $T$ and $\mathbf{a}$. Analogously to the first part $\operatorname{div} \mathbf{A} \in L^{2}\left(0, T ; H_{0}^{1}(\Omega)\right)^{d}$. Hence $E_{T} \mathbf{a}:=\mathbf{A}-B(\operatorname{div} \mathbf{A}) \in L^{2}\left(0, T ; H_{0}^{2}(\Omega)\right)^{d}$. Moreover, due to [18, Theorem 2.5] we also have

$$
\|B(\operatorname{div} \mathbf{A})\|_{H^{1}\left(0, T ; L^{2}(\Omega)\right)} \leq C\left\|\operatorname{div}_{n} \mathbf{A}\right\|_{H^{1}\left(0, T ; H_{(0)}^{-1}(\Omega)\right)} \leq C^{\prime}\|\mathbf{A}\|_{H^{1}\left(0, T ; L^{2}(\Omega)\right)},
$$

where $C, C^{\prime}>0$ are independent of $T$. Altogether $E_{T}$ has the stated properties.

\section{Short Time Existence of Strong Solutions}

In this section we prove existence of strong and unique solutions of system (1.6)-(1.10) and (1.12)-(1.14) locally in time in the case $a(c) \equiv m(c) \equiv 1$, i.e., prove Theorem 1.2 As noted before we will assume that $\beta \neq 0$ since in the case $\beta=0$ the linearized system is completely different and short time existence of strong solutions is known in that case, cf. e.g. [4]. In this case we can eliminate the generalized pressure $g_{0}$ and the chemical potential $\mu$ as follows:

First of all, because of (1.11), one easily calculates that

$$
\frac{\partial \rho}{\partial c}=-\beta \rho^{2}, \quad \frac{\partial(\rho c)}{\partial c}=\rho+\frac{\partial \rho}{\partial c} c=\alpha \rho^{2} .
$$

For the following let $c$ be a sufficiently smooth solution such that $|c(t, x)| \leq 1+\varepsilon_{0}$, where $\varepsilon_{0}>0$ is as in Assumption 1.1. Then (1.7) and (3.1) imply

$$
-\beta \rho^{2}\left(\partial_{t} c+\mathbf{v} \cdot \nabla c\right)=-\rho \operatorname{div} \mathbf{v}
$$

Combining this with (1.8), we obtain the simple identity

$$
\operatorname{div} \mathbf{v}=\beta \Delta \mu_{0}
$$


Thus $\mu_{0}=\beta^{-1} \Delta_{N}^{-1} \operatorname{div} \mathbf{v}=\beta^{-1} G(\operatorname{div} \mathbf{v})$ since $\left.\mathbf{n} \cdot \nabla \mu_{0}\right|_{\partial \Omega}=0$, where $G(g)$ is defined by

$$
\begin{array}{cl}
\Delta G(g)=g & \text { in } \Omega, \\
\partial_{n} G(g)=0 & \text { on } \partial \Omega,
\end{array}
$$

and $\int_{\Omega} G(g) d x=0$. Note that this implies

$$
\nabla G(\operatorname{div} \mathbf{v})=\left(I-P_{\sigma}\right) \mathbf{v} .
$$

Using this and (1.9), we can eliminate $g_{0}, \mu_{0}$ and $\bar{p}(t)$ from the system (1.6)-(1.9) and obtain the equivalent system

$$
\begin{array}{rlrl}
\partial_{t} \mathbf{v}+\mathbf{v} \cdot \nabla \mathbf{v}-\rho^{-1} \operatorname{div} \mathbb{S}(c, \mathbb{D} \mathbf{v}) & & \\
+\frac{1}{\beta} \nabla\left(\rho^{-2}(\Delta c-\phi(c))\right) & =\frac{1}{\beta} G(\operatorname{div} \mathbf{v}) \nabla c-\frac{1}{\beta^{2}} \nabla\left(\rho^{-1} G(\operatorname{div} \mathbf{v})\right) & & \text { in } Q_{T} \\
\rho \partial_{t} c+\rho \mathbf{v} \cdot \nabla c & =\beta^{-1} \operatorname{div} \mathbf{v} . & & \text { in } Q_{T}
\end{array}
$$

together with

$$
\begin{aligned}
\left.\mathbf{n} \cdot \mathbf{v}\right|_{\partial \Omega}=(\mathbf{n} \cdot \mathbb{S}(c, \mathbb{D} \mathbf{v}))_{\tau}+\left.\gamma(c) \mathbf{v}_{\tau}\right|_{\partial \Omega}=\left.\partial_{n} c\right|_{\partial \Omega} & =0 & & \text { on } S_{T}, \\
\left.(\mathbf{v}, c)\right|_{t=0} & =\left(\mathbf{v}_{0}, c_{0}\right) & & \text { in } \Omega .
\end{aligned}
$$

This is indeed an equivalent system since, if $(\mathbf{v}, c)$ solve the system above, we can simply define $g_{0}$ and $\bar{p}(t)$ by the equation (1.9) and $\mu_{0}$ by $\mu_{0}=\beta^{-1} G(\operatorname{div} \mathbf{v})$. Then $\left.\mathbf{n} \cdot \nabla \mu_{0}\right|_{\partial \Omega}$ is automatically satisfied.

We will construct strong solutions by linearizing the system, proving that the associated linear operator is an isomorphism between suitable $L^{2}$-Sobolev spaces, and applying the contraction mapping principle to prove existence and uniqueness of the full system for sufficiently small times.

To this end, let $\tilde{c}_{0} \in H^{1}\left(0, T_{0} ; H^{1}(\Omega)\right) \cap L^{2}\left(0, T_{0} ; H^{3}(\Omega) \cap H_{N}^{2}(\Omega)\right)$ be such that $\left.\tilde{c}_{0}\right|_{t=0}=c_{0}$. The existence of such an $\tilde{c}_{0}$ follows from Theorem 3.1 below. Then (3.7)-(3.10) are equivalent to

$$
L(c)\left(\begin{array}{c}
\mathbf{v} \\
\rho\left(c-\tilde{c}_{0}\right)
\end{array}\right)=\mathcal{F}(\mathbf{v}, c),
$$

where for given $c$ the linear operator $L(c): X_{T} \rightarrow Y_{T}$ is defined by

$$
L(c)\left(\begin{array}{c}
\mathbf{v} \\
c^{\prime}
\end{array}\right)=\left(\begin{array}{c}
\partial_{t} \mathbf{v}-\operatorname{div} \widetilde{\mathbb{S}}(c, \mathbb{D} \mathbf{v})+\frac{1}{\beta \alpha} \nabla \operatorname{div}\left(\rho^{-4} \nabla c^{\prime}\right) \\
\partial_{t} c^{\prime}-\beta^{-1} \operatorname{div} \mathbf{v} \\
\left(\mathbf{n} \cdot \widetilde{\mathbb{S}}\left(c, \mathbb{D}\left(P_{\sigma} \mathbf{v}\right)\right)\right)_{\tau}+\left.\tilde{\gamma}(c)\left(P_{\sigma} \mathbf{v}\right)_{\tau}\right|_{\partial \Omega} \\
\left.(\mathbf{v}, c)\right|_{t=0}
\end{array}\right), \quad\left(\begin{array}{c}
\mathbf{v} \\
c^{\prime}
\end{array}\right) \in X_{T}
$$


and $c^{\prime}$ corresponds to $\rho c$. Here we have used $\nabla(\rho c)=\alpha \rho^{2} \nabla c$ and set $\widetilde{\mathbb{S}}(c, \mathbb{D} \mathbf{v})=$ $2 \tilde{\nu}(c) \mathbb{D} \mathbf{v}+\tilde{\eta}(c) \operatorname{div} \mathbf{v} \mathbb{I}, \tilde{\nu}(c)=\hat{\rho}(c)^{-1} \nu(c), \tilde{\eta}(c)=\hat{\rho}(c)^{-1} \eta(c)$, and $\tilde{\gamma}(c)=\hat{\rho}(c)^{-1} \gamma(c)$. Moreover, $\mathcal{F}: X_{T} \rightarrow Y_{T}$ is a non-linear mapping defined by

$$
\begin{aligned}
\mathcal{F}(\mathbf{v}, c)= & \left(\begin{array}{c}
F_{1}(\mathbf{v}, c) \\
-\rho \mathbf{v} \cdot \nabla c-\rho \partial_{t} \tilde{c}_{0}+\partial_{t} \rho\left(c-\tilde{c}_{0}\right) \\
\left(\mathbf{n} \cdot \widetilde{\mathbb{S}}\left(c, \nabla^{2} G(\operatorname{div} \mathbf{v})\right)\right)_{\tau}+\left.\tilde{\gamma}(c) \nabla_{\tau} G(\operatorname{div} \mathbf{v})\right|_{\partial \Omega} \\
\left(\mathbf{v}_{0}, 0\right)
\end{array}\right), \\
F_{1}(\mathbf{v}, c)= & \frac{G(\operatorname{div} \mathbf{v}) \nabla c}{\beta}+\frac{1}{\beta} \nabla\left(\frac{\phi(c)}{\rho^{2}}-\frac{G(\operatorname{div} \mathbf{v})}{\beta^{2} \rho}-\left[\rho^{-2}, \operatorname{div}\right] \nabla c\right) \\
& +\frac{1}{\beta} \nabla\left(\rho^{-2} \Delta c_{0}\right)-\mathbf{v} \cdot \nabla \mathbf{v}-\nabla \rho^{-1} \cdot \mathbb{S}(c, \mathbb{D} \mathbf{v}),
\end{aligned}
$$

and $X_{T}=X_{T}^{1} \times X_{T}^{2}$,

$$
\begin{aligned}
X_{T}^{1} & =\left\{\mathbf{u} \in H^{1}\left(0, T ; L^{2}(\Omega)^{d}\right) \cap L^{2}\left(0, T ; H^{2}(\Omega)^{d}\right):\left.\mathbf{n} \cdot \mathbf{u}\right|_{\partial \Omega}=0\right\}, \\
X_{T}^{2} & =\left\{c^{\prime} \in H^{1}\left(0, T ; H^{1}(\Omega)\right) \cap L^{2}\left(0, T ; H^{3}(\Omega)\right):\left.c^{\prime}\right|_{t=0}=0,\left.\mathbf{n} \cdot \nabla c^{\prime}\right|_{\partial \Omega}=0\right\}, \\
Y_{T} & =L^{2}\left(Q_{T}\right)^{d} \times L^{2}\left(0, T ; H_{0}^{1}(\Omega)\right) \times\left\{\mathbf{a} \in H^{\frac{1}{4}, \frac{1}{2}}\left(S_{T}\right): \mathbf{a}_{n}=0\right\} \times H_{n}^{1}(\Omega) \times H_{N}^{2}(\Omega),
\end{aligned}
$$

where $H^{\frac{1}{4}}, \frac{1}{2}\left(S_{T}\right):=H^{\frac{1}{4}}\left(0, T ; L^{2}(\partial \Omega)\right) \cap L^{2}\left(0, T ; H^{\frac{1}{2}}(\partial \Omega)\right)$ Here $[A, B]=A B-B A$ denotes the commutator of two operators. The spaces $X_{T}^{1}, X_{T}^{2}$, and $Y_{T}$ are normed by

$$
\begin{aligned}
\|\mathbf{v}\|_{X_{T}^{1}} & =\left\|\left(\partial_{t} \mathbf{v}, \nabla^{2} \mathbf{v}\right)\right\|_{L^{2}\left(Q_{T}\right)}+\left\|\left.\mathbf{v}\right|_{t=0}\right\|_{H^{1}(\Omega)}, \\
\left\|c^{\prime}\right\|_{X_{T}^{2}} & =\left\|\left(c^{\prime}, \partial_{t} c^{\prime}, \partial_{t} \nabla c^{\prime}, \nabla^{3} c^{\prime}\right)\right\|_{L^{2}\left(Q_{T}\right)}+\left\|\left.c^{\prime}\right|_{t=0}\right\|_{H^{2}(\Omega)}, \\
\left\|\left(\mathbf{f}, g, \mathbf{a}, \mathbf{v}_{0}\right)\right\|_{Y_{T}} & =\|(\mathbf{f}, \nabla g)\|_{L^{2}\left(Q_{T}\right)}+\|\mathbf{a}\|_{H^{\frac{1}{4}, \frac{1}{2}\left(S_{T}\right)}}+\left\|\mathbf{v}_{0}\right\|_{H^{1}(\Omega)}+\left\|c_{0}\right\|_{H^{2}(\Omega)} .
\end{aligned}
$$

In order to apply the contraction mapping principle to (3.11) for sufficiently small $T>0$, it is essential that $L\left(c_{0}\right)$ is an isomorphism:

THEOREM 3.1 Let $c_{0} \in H^{2}(\Omega)$, let $T_{0}>0$, and let Assumption 1.1 hold true. Then $L\left(c_{0}\right): X_{T} \rightarrow Y_{T}$ is an isomorphism for every $0<T \leq T_{0}$ and there is a constant $C\left(T_{0}\right)>0$ such that

$$
\left\|L\left(c_{0}\right)^{-1}\right\|_{\mathcal{L}\left(Y_{T}, X_{T}\right)} \leq C\left(T_{0}\right) \quad \text { for all } 0<T \leq T_{0} .
$$

The proof of this theorem is postponed to Section 4. The second ingredient for the application of the contraction mapping to (3.11) is the fact that $\mathcal{F}: X_{T} \rightarrow Y_{T}$ is locally Lipschitz continuous with arbitrarily small Lipschitz constant if $T>0$ is sufficiently small:

Proposition 3.2 Let $R>0$ and let Assumption 1.1 be satisfied. Then there is a constant $C(T, R)>0$ such that

$$
\left\|\mathcal{F}\left(\mathbf{v}_{1}, c_{1}\right)-\mathcal{F}\left(\mathbf{v}_{2}, c_{2}\right)\right\|_{Y_{T}} \leq C(T, R)\left\|\left(\mathbf{v}_{1}-\mathbf{v}_{2}, c_{1}-c_{2}\right)\right\|_{X_{T}}
$$


for all $\left(\mathbf{v}_{j}, c_{j}\right) \in X_{T}$ with $\left\|\left(\mathbf{v}_{j}, c_{j}\right)\right\|_{X_{T}} \leq R$ and $\left.c_{j}\right|_{t=0}=c_{0}$, where $j=1,2$. Moreover, $C(T, R) \rightarrow 0$ as $T \rightarrow 0$.

Proof: Let $F_{2}(\mathbf{v}, c)=-\rho \mathbf{v} \cdot \nabla c$ and let $F_{3}(\mathbf{v}, c)=-\partial_{t} \rho\left(c-c_{0}\right)$. For $F^{\prime}(\mathbf{v}, c)=$ $\left(F_{1}(\mathbf{v}, c), F_{2}(\mathbf{v}, c)\right)$ we will show that

$$
\left\|F^{\prime}\left(\mathbf{v}_{1}, c_{1}\right)-F^{\prime}\left(\mathbf{v}_{2}, c_{2}\right)\right\|_{L^{p}\left(0, T ; L^{2}(\Omega)^{d} \times H^{1}(\Omega)\right)} \leq C\left(p, R, T_{0}\right)\left\|\left(\mathbf{v}_{1}-\mathbf{v}_{2}, c_{1}-c_{2}\right)\right\|_{X_{T}}
$$

for all $0<T \leq T_{0}$ and for some $p>2$. (Note that the third component of $\mathcal{F}$ is constant.) Then the statement of the proposition for these terms follows from the estimate

$$
\|f\|_{L^{2}(0, T ; X)} \leq T^{\frac{1}{2}-\frac{1}{p}}\|f\|_{L^{p}(0, T ; X)},
$$

where $X$ is an arbitrary Banach space. In order to estimate the terms involving $c$, we use that

$$
\|c\|_{L^{\infty}\left(0, T ; H^{2}(\Omega)\right)} \leq C\|c\|_{X_{T}^{2}}
$$

due to (2.4) and (2.1), where $C$ is independent of $T>0$. Since $H^{2}(\Omega)$ is an algebra with respect to pointwise multiplication, we have $\left.\widetilde{F}(c) \in L^{\infty}\left(0, T ; H^{2}(\Omega)\right)\right)$ for all $\widetilde{F} \in C^{3}(\mathbb{R}), c \in X_{T}^{2}$, as well as

$$
\left\|\widetilde{F}\left(c_{1}\right)-\widetilde{F}\left(c_{2}\right)\right\|_{L^{\infty}\left(0, T ; H^{2}(\Omega)\right)} \leq C(R, \widetilde{F})\left\|c_{1}-c_{2}\right\|_{X_{T}^{2}}
$$

for all $c_{j} \in X_{T}^{2}$ with $\left\|c_{j}\right\|_{X_{T}^{2}} j=1,2$. Hence

$$
\left\|\nabla\left(\hat{\rho}\left(c_{1}\right)^{-2} \phi\left(c_{1}\right)-\hat{\rho}\left(c_{2}\right)^{-2} \phi\left(c_{2}\right)\right)\right\|_{L^{\infty}\left(0, T ; H^{1}(\Omega)\right)} \leq C(R, \phi, \hat{\rho})\left\|c_{1}-c_{2}\right\|_{X_{T}^{2}}
$$

for all $c_{j} \in X_{T}^{2}$ with $\left\|c_{j}\right\|_{X_{T}^{2}} \leq R, j=1,2$. Moreover,

$$
\|G(\operatorname{div} \mathbf{v})\|_{L^{\infty}\left(0, T ; H^{2}(\Omega)\right)} \leq C\|\mathbf{v}\|_{L^{\infty}\left(0, T ; H^{1}(\Omega)\right)} \leq C^{\prime}\|\mathbf{v}\|_{X_{T}^{1}}
$$

for constants $C, C^{\prime}$ independent of $T>0$ due to (2.9) and (2.4). Since the product of Lipschitz continuous functions is again Lipschitz continuous, it is sufficient to verify that all the products appearing in $F(v, c)$ are well-defined, which is done as follows:

$$
\begin{aligned}
\|G(\operatorname{div} \mathbf{v}) \nabla c\|_{L^{\infty}\left(0, T ; L^{2}(\Omega)\right)} & \leq C\|G(\operatorname{div} \mathbf{v})\|_{L^{\infty}\left(0, T ; H^{2}(\Omega)\right)}\|c\|_{L^{\infty}\left(0, T ; H^{2}(\Omega)\right)} \leq C(R) \\
\left\|\nabla\left(\rho^{-1} G(\operatorname{div} \mathbf{v})\right)\right\|_{L^{\infty}\left(0, T ; L^{2}(\Omega)\right)} & \leq C\|G(\operatorname{div} \mathbf{v})\|_{L^{\infty}\left(0, T ; H^{2}(\Omega)\right)}\left\|\rho^{-1}\right\|_{L^{\infty}\left(0, T ; H^{2}(\Omega)\right)} \leq C(R) \\
\|\mathbf{v} \cdot \nabla \mathbf{v}\|_{L^{4}\left(0, T ; L^{2}(\Omega)\right)} & \leq\|\mathbf{v}\|_{L^{\infty}\left(0, T ; L^{6}(\Omega)\right)}\|\nabla \mathbf{v}\|_{L^{4}\left(0, T ; L^{3}(\Omega)\right)} \\
& \leq C(R)\|\mathbf{v}\|_{L^{\infty}\left(0, T ; H^{1}(\Omega)\right)}^{\frac{1}{2}}\|\nabla \mathbf{v}\|_{L^{2}\left(0, T ; L^{6}(\Omega)\right)}^{\frac{1}{2}} \leq C^{\prime}(R) \\
\left\|\nabla \rho^{-1} \cdot \mathbb{S}(c, \mathbb{D} \mathbf{v})\right\|_{L^{4}\left(0, T ; L^{2}(\Omega)\right)} & \leq C\left\|\nabla \rho^{-1}\right\|_{L^{\infty}\left(0, T ; L^{6}\right)}\|(\nu(c), \eta(c))\|_{L^{\infty}\left(Q_{T}\right)}\|\nabla \mathbf{v}\|_{L^{4}\left(0, T ; L^{3}\right)} \\
& \leq C(R)\|\nabla \mathbf{v}\|_{L^{\infty}\left(0, T ; L^{2}\right)}^{\frac{1}{2}}\|\nabla v\|_{L^{2}\left(0, T ; L^{6}\right)}^{\frac{1}{2}} \leq C^{\prime}(R) \\
\|\rho \mathbf{v} \cdot \nabla c\|_{L^{4}\left(0, T ; H^{1}(\Omega)\right)} & \leq C\|\mathbf{v}\|_{L^{4}\left(0, T ; B_{3,1}^{1}(\Omega)\right)}\|\nabla r(c)\|_{L^{\infty}\left(0, T ; H^{1}\right)} \\
& \leq C\|\mathbf{v}\|_{L^{2}\left(0, T ; H^{2}\right)}^{\frac{1}{2}}\|\mathbf{v}\|_{L^{\infty}\left(0, T ; H^{1}\right)}^{\frac{1}{2}}\|\nabla r(c)\|_{L^{\infty}\left(0, T ; H^{1}\right)} \\
& \leq C(R),
\end{aligned}
$$


for all $(\mathbf{v}, c) \in X_{T}$ with $\|(\mathbf{v}, c)\|_{X_{T}} \leq R$, where $r^{\prime}(c)=\hat{\rho}(c)$ and we have used (2.2) and

$$
\left(H^{1}(\Omega), H^{2}(\Omega)\right)_{\frac{1}{2}, 1}=B_{2,1}^{\frac{3}{2}}(\Omega) \hookrightarrow B_{3,1}^{1}(\Omega) .
$$

In order to estimate $\nabla\left(\left[\rho^{-2}, \operatorname{div}\right] \nabla c\right)$, we use that $\nabla\left[\rho^{-2}, \operatorname{div}\right] \nabla c=-\nabla\left(\nabla \rho^{-2} \cdot \nabla c\right)=$ $\nabla^{2} \rho^{-2} \cdot \nabla c+\nabla \rho^{-2} \cdot \nabla^{2} c$ and

$$
\begin{aligned}
\left\|\nabla^{2} \rho^{-2} \cdot \nabla c\right\|_{L^{4}\left(0, T ; L^{2}(\Omega)\right)} & \leq C\|\rho\|_{L^{\infty}\left(0, T ; H^{2}(\Omega)\right)}\|\nabla c\|_{L^{4}\left(0, T ; L^{\infty}(\Omega)\right)} \\
& \leq C(R)\|\nabla c\|_{L^{2}\left(0, T ; H^{2}(\Omega)\right)}\|\nabla c\|_{L^{\infty}\left(0, T ; H^{1}(\Omega)\right)} \leq C^{\prime}(R)
\end{aligned}
$$

due to $\|f\|_{L^{\infty}(\Omega)} \leq C\|f\|_{B_{2,1}^{\frac{3}{2}}(\Omega)} \leq C^{\prime}\|f\|_{H^{1}}^{\frac{1}{2}}\|f\|_{H^{2}}^{\frac{1}{2}}$. The same estimate holds true for $\nabla \rho^{-2} \cdot \nabla^{2} c$. Hence (3.13) holds with $p=4$.

Furthermore, we estimate $F_{3}(\mathbf{v}, c)$ as follows

$$
\begin{aligned}
& \left\|\left(\hat{\rho}\left(c_{1}\right)-\hat{\rho}\left(c_{2}\right)\right) \partial_{t} \tilde{c}_{0}\right\|_{L^{2}\left(0, T ; H^{1}(\Omega)\right)} \\
& \quad \leq C\left\|\hat{\rho}\left(c_{1}\right)-\hat{\rho}\left(c_{2}\right)\right\|_{L^{\infty}\left(0, T ; B_{2,1}^{3 / 2}(\Omega)\right)}\left\|\partial_{t} \tilde{c}_{0}\right\|_{L^{2}\left(0, T ; H^{1}(\Omega)\right)} \leq C\left(R, \tilde{c}_{0}\right) T^{\frac{1}{4}}\left\|c_{1}-c_{2}\right\|_{X_{T}^{2}} \\
& \left\|\left(\partial_{t} \hat{\rho}\left(c_{1}\right)-\partial_{t} \hat{\rho}\left(c_{2}\right)\right)\left(c_{1}-\tilde{c}_{0}\right)\right\|_{L^{2}\left(0, T ; H^{1}(\Omega)\right)} \\
& \quad \leq C\left\|\partial_{t} \hat{\rho}\left(c_{1}\right)-\partial_{t} \hat{\rho}\left(c_{2}\right)\right\|_{L^{2}\left(0, T ; H^{1}\right)}\left\|c_{1}-\tilde{c}_{0}\right\|_{L^{\infty}\left(0, T ; B_{2,1}^{3 / 2}\right)} \leq C\left(R, \tilde{c}_{0}\right) T^{\frac{1}{4}}\left\|c_{1}-c_{2}\right\|_{X_{T}^{2}} \\
& \left\|\partial_{t} \hat{\rho}\left(c_{2}\right)\left(c_{1}-c_{2}\right)\right\|_{L^{2}\left(0, T ; H^{1}(\Omega)\right)} \\
& \quad \leq C\left\|\partial_{t} \rho\left(c_{2}\right)\right\|_{L^{2}\left(0, T ; H^{1}(\Omega)\right)}\left\|c_{1}-c_{2}\right\|_{L^{\infty}\left(0, T ; B_{2,1}^{3 / 2}(\Omega)\right)} \leq C\left(R, \tilde{c}_{0}\right) T^{\frac{1}{4}}\left\|c_{1}-c_{2}\right\|_{X_{T}^{2}}
\end{aligned}
$$

since

$$
X_{T}^{2} \hookrightarrow C^{\frac{1}{2}}\left([0, T] ; H^{1}(\Omega)\right) \cap L^{\infty}\left(0, T ; H^{2}(\Omega)\right) \hookrightarrow C^{\frac{1}{8}}\left([0, T] ; H^{\frac{7}{4}}(\Omega)\right),
$$

$H^{\frac{7}{4}}(\Omega) \hookrightarrow B_{2,1}^{\frac{3}{2}}(\Omega)$, and $\left.\left(c_{1}-c_{2}\right)\right|_{t=0}=\left.\left(c_{1}-\tilde{c}_{0}\right)\right|_{t=0}=0$.

Finally, it remains to estimate the third component of $\mathcal{F}(\mathbf{v}, c)$. To this end we use that

$$
\begin{aligned}
\|\left(\mathbf{n} \cdot \nabla^{2} G(\operatorname{div} \mathbf{v})_{\tau} \|_{L^{2}\left(0, T ; H^{\frac{3}{2}}(\partial \Omega)\right)}\right. & \leq C\|\mathbf{v}\|_{L^{2}\left(0, T ; H^{2}(\Omega)\right)} \\
\|\left(\mathbf{n} \cdot \nabla^{2} G(\operatorname{div} \mathbf{v})_{\tau} \|_{H^{1}\left(0, T ; H^{-\frac{1}{2}}(\partial \Omega)\right)}\right. & \leq C\|\mathbf{v}\|_{H^{1}\left(0, T ; L^{2}(\Omega)\right)}
\end{aligned}
$$

for all $\mathbf{v} \in X_{T}^{1}$ since $\left(\mathbf{n} \cdot \nabla^{2} G(\operatorname{div} \mathbf{v})\right)_{\tau}=A \gamma_{0} \mathbf{v}$ for some first order tangential differential operator $A$, cf. Lemma 2.2. Hence

$$
\begin{aligned}
& \left\|\left(\mathbf{n} \cdot \nabla^{2} G(\operatorname{div} \mathbf{v})\right)_{\tau}\right\|_{L^{2}\left(0, T ; H^{\frac{1}{2}}(\partial \Omega)\right)} \\
& \quad \leq T^{\frac{1}{2}} \|\left(\mathbf{n} \cdot \nabla^{2} G(\operatorname{div} \mathbf{v})\left\|_{B U C\left([0, T] ; H^{\frac{1}{2}}(\partial \Omega)\right)} \leq C T^{\frac{1}{2}}\right\| \mathbf{v} \|_{X_{T}^{1}}\right.
\end{aligned}
$$

for all $\mathbf{v} \in X_{T}^{1}$ due to (2.4) and

$$
\|\left(\mathbf { n } \cdot \nabla ^ { 2 } G ( \operatorname { d i v } \mathbf { v } ) _ { \tau } \| _ { H ^ { \frac { 1 } { 4 } ( 0 , T ; L ^ { 2 } ( \partial \Omega ) ) } } \leq T ^ { \frac { 1 } { 2 } } \| \left(\mathbf{n} \cdot \nabla^{2} G(\operatorname{div} \mathbf{v})\left\|_{C^{\frac{1}{3}\left([0, T] ; L^{2}(\partial \Omega)\right)}} \leq C T^{\frac{1}{2}}\right\| \mathbf{v} \|_{X_{T}^{1}}\right.\right.
$$


due to $(2.3)$ and since $B U C\left([0, T] ; H^{1}\right) \cap C^{\frac{1}{2}}\left([0, T] ; H^{-\frac{1}{2}}\right) \hookrightarrow C^{\frac{1}{3}}\left([0, T] ; L^{2}\right)$ because of $\|f\|_{L^{2}} \leq C\|f\|_{H^{1}}^{\frac{1}{3}}\|f\|_{H^{-\frac{1}{2}}}^{\frac{2}{3}}$. Together we have

$$
\|\left(\mathbf{n} \cdot \nabla^{2} G(\operatorname{div} \mathbf{v})_{\tau}\left\|_{H^{\frac{1}{4}, \frac{1}{2}}\left(S_{T}\right)} \leq C T^{\frac{1}{2}}\right\| \mathbf{v} \|_{X_{T}^{1}}\right.
$$

By the same arguments one shows that

$$
\left\|(\nabla G(\operatorname{div} \mathbf{v}))_{\tau}\right\|_{H^{\frac{1}{4}, \frac{1}{2}\left(S_{T}\right)}} \leq C T^{\frac{1}{2}}\|\mathbf{v}\|_{X_{T}^{1}}
$$

Moreover, using (13.16) $),\|f g\|_{H^{\frac{1}{2}(\partial \Omega)}} \leq C\|f\|_{B_{2,1}^{1}(\partial \Omega)}\|g\|_{H^{\frac{1}{2}(\partial \Omega)}}$ as well as $\|f g\|_{L^{2}(\partial \Omega)} \leq$ $\|f\|_{L^{4}(\partial \Omega)}\|g\|_{L^{4}(\partial \Omega)} \leq C\|f\|_{H^{\frac{1}{2}(\partial \Omega)}}\|g\|_{H^{\frac{1}{2}(\partial \Omega)}}$, one obtains

$$
\begin{aligned}
& \left\|\left(\tilde{\nu}\left(c_{1}\right)-\tilde{\nu}\left(c_{2}\right)\right) a\right\|_{H^{\frac{1}{4}}\left(0, T ; L^{2}(\partial \Omega)\right)} \\
& \leq C T^{\frac{1}{4}}\left\|\left(\tilde{\nu}\left(c_{1}\right)-\tilde{\nu}\left(c_{2}\right)\right)\right\|_{C^{\frac{1}{2}\left([0, T] ; H^{\frac{1}{2}}(\partial \Omega)\right)}}\|a\|_{L^{2}\left(0, T ; H^{\frac{1}{2}}(\partial \Omega)\right)} \\
& +C T^{\frac{1}{4}}\left\|\left(\tilde{\nu}\left(c_{1}\right)-\tilde{\nu}\left(c_{2}\right)\right)\right\|_{C^{\frac{1}{4}\left([0, T] ; B_{2,1}^{1}(\partial \Omega)\right)}}\|a\|_{H^{\frac{1}{4}\left(0, T ; L^{2}(\partial \Omega)\right)}}
\end{aligned}
$$

and therefore

$$
\begin{aligned}
& \left\|\left(\tilde{\nu}\left(c_{1}\right)-\tilde{\nu}\left(c_{2}\right)\right) a\right\|_{H^{\frac{1}{4}, \frac{1}{2}}\left(S_{T}\right)} \\
& \quad \leq C(R, \tilde{\nu}) T^{\frac{1}{4}}\left\|c_{1}-c_{2}\right\|_{B U C\left([0, T] ; H^{2}(\Omega)\right) \cap C^{\frac{1}{2}\left([0, T] ; H^{1}(\Omega)\right)}}\|a\|_{H^{\frac{1}{4}, \frac{1}{2}}\left(S_{T}\right)}
\end{aligned}
$$

for all $a \in H^{\frac{1}{4}, \frac{1}{2}}\left(S_{T}\right)$ and $c_{j} \in X_{T}^{2}$ with $\left\|c_{j}\right\|_{X_{T}^{2}} \leq R$ and $\left.c_{j}\right|_{t=0}=c_{0}, j=1,2$. Combining these estimates, we obtain

$$
\begin{aligned}
&\left\|\left(\tilde{\nu}\left(c_{1}\right) \mathbf{n} \cdot \nabla^{2} G\left(\operatorname{div} \mathbf{v}_{1}\right)\right)_{\tau}-\left(\tilde{\nu}\left(c_{2}\right) \mathbf{n} \cdot \nabla^{2} G\left(\operatorname{div} \mathbf{v}_{2}\right)\right)_{\tau}\right\|_{H^{\frac{1}{4}, \frac{1}{2}}\left(S_{T}\right)} \\
& \leq\left\|\left(\left(\tilde{\nu}\left(c_{1}\right)-\tilde{\nu}\left(c_{2}\right)\right) \mathbf{n} \cdot \nabla^{2} G\left(\operatorname{div} \mathbf{v}_{1}\right)\right)_{\tau}\right\|_{H^{\frac{1}{4}, \frac{1}{2}}\left(S_{T}\right)} \\
&+\|\left(\tilde{\nu}\left(c_{2}\right) \mathbf{n} \cdot \nabla^{2} G\left(\operatorname{div}\left(\mathbf{v}_{1}-\mathbf{v}_{2}\right)\right)_{\tau} \|_{H^{\frac{1}{4}, \frac{1}{2}}\left(S_{T}\right)}\right. \\
& \leq C(R) T^{\frac{1}{4}}\left(\left\|\mathbf{v}_{1}-\mathbf{v}_{2}\right\|_{X_{T}^{1}}+\left\|c_{1}-c_{2}\right\|_{X_{T}^{2}}\right)
\end{aligned}
$$

for all $u_{j}=\left(\mathbf{v}_{j}, c_{j}\right) \in X_{T}$ with $\left\|u_{j}\right\|_{X_{T}} \leq R$ and $\left.c_{j}\right|_{t=0}=c_{0}, j=1,2$ since $\|(\nabla \mathbf{v}, \mathbf{v})\|_{H^{\frac{1}{4}, \frac{1}{2}\left(S_{T}\right)}} \leq C\|\mathbf{v}\|_{X_{T}^{1}}$. In the same way one can estimate $\tilde{\gamma}\left(c_{1}\right)\left(\nabla G\left(\operatorname{div} \mathbf{v}_{1}\right)_{\tau}-\right.$ $\tilde{\gamma}\left(c_{2}\right)\left(\left.\nabla G\left(\operatorname{div} \mathbf{v}_{2}\right)_{\tau}\right|_{\partial \Omega}\right.$, which proves the necessary estimate of the third component of $\mathcal{F}$.

Altogether this proves the proposition.

Combining Theorem 3.1 and Proposition 3.2, we are now able to prove our main result.

Proof of Theorem 1.2: First of all, let $\tilde{c}_{0} \in X_{1}^{2}$ be such that $\left\|\tilde{c}_{0}\right\|_{X_{1}^{2}} \leq C^{\prime}\left\|c_{0}\right\|_{H^{2}(\Omega)}$ for some fixed constant $C^{\prime}>0$ and let

$$
R=\max \left(C^{\prime}\left\|c_{0}\right\|_{H^{2}(\Omega)},\left\|S\left(\tilde{c}_{0}\right) L^{-1}\left(\tilde{c}_{0}\right) \mathcal{F}(0)\right\|_{X_{T}}\right)
$$


where $S$ is defined below. We will construct a solution in $B_{R}(0) \subset X_{T}$ for sufficiently small $0<T \leq 1$ with the aid of the contraction mapping principle. If

$$
c_{1}, c_{2} \in B_{R}(0) \subset B U C\left([0, T] ; H^{2}(\Omega)\right) \cap C^{\frac{1}{2}}\left(0, T ; H^{1}(\Omega)\right)=: \widetilde{X}_{T}^{2},
$$

$0<T \leq 1$ with $\left.c_{1}\right|_{t=0}=\left.c_{2}\right|_{t=0}$, we conclude

$$
\begin{aligned}
\| L\left(c_{1}\right) u- & L\left(c_{2}\right) u \|_{Y_{T}} \\
\leq & C\left(\left\|\widetilde{\mathbb{S}}\left(c_{1}, \mathbb{D} \mathbf{v}\right)-\widetilde{\mathbb{S}}\left(c_{2}, \mathbb{D} \mathbf{v}\right)\right\|_{L^{2}\left(0, T ; H^{1}\right)}+\left\|\widetilde{\mathbb{S}}\left(c_{1}, \mathbb{D} \mathbf{v}\right)-\left.\widetilde{\mathbb{S}}\left(c_{2}, \mathbb{D} \mathbf{v}\right)\right|_{\partial \Omega}\right\|_{H^{\frac{1}{4}, \frac{1}{2}\left(S_{T}\right)}}\right. \\
& \left.+\left\|\left.\left(\tilde{\gamma}\left(c_{1}\right)-\tilde{\gamma}\left(c_{2}\right)\right) \mathbf{v}\right|_{\partial \Omega}\right\|_{H^{\frac{1}{4}, \frac{1}{2}\left(S_{T}\right)}}+\left\|\left(\hat{\rho}\left(c_{1}\right)^{-2}-\hat{\rho}\left(c_{2}\right)^{-2}\right) \nabla c^{\prime}\right\|_{L^{2}\left(0, T ; H^{2}\right)}\right) \\
\leq & C\left(\left\|c_{1}-c_{2}\right\|_{L^{\infty}\left(0, T ; B_{3,1}^{1}\right)}\|\mathbf{v}\|_{X_{T}^{1}}+\left\|\hat{\rho}\left(c_{1}\right)^{-2}-\hat{\rho}\left(c_{2}\right)^{-2}\right\|_{L^{\infty}\left(0, T ; H^{2}\right)}\left\|\nabla c^{\prime}\right\|_{L^{2}\left(0, T ; B_{3,1}^{1}\right)}\right. \\
& \left.+\left\|\hat{\rho}\left(c_{1}\right)^{-2}-\hat{\rho}\left(c_{2}\right)^{-2}\right\|_{L^{\infty}\left(0, T ; B_{3,1}^{1}\right)}\left\|c^{\prime}\right\|_{L^{2}\left(0, T ; H^{3}\right)}\right) \\
\leq & C(R)\left(T^{\frac{1}{4}}\left\|c_{1}-c_{2}\right\|_{\tilde{X}_{T}^{2}}\|u\|_{X_{T}}+T^{\frac{1}{4}}\left\|c_{1}-c_{2}\right\|_{B U C\left([0, T] ; H^{2}\right)}\left\|c^{\prime}\right\|_{L^{4}\left([0, T] ; B_{2,1}^{\left.\frac{5}{2}\right)}\right)}\right) \\
\leq & C(R) T^{\frac{1}{4}}\left(\left\|c_{1}-c_{2}\right\|_{C^{\frac{1}{2}\left([0, T] ; H^{1}\right)}}+\left\|c_{1}-c_{2}\right\|_{B U C\left([0, T] ; H^{2}\right)}\right)\|u\|_{X_{T} .}
\end{aligned}
$$

where $u=\left(\mathbf{v}, c^{\prime}\right) \in X_{T}$ with $\left.c^{\prime}\right|_{t=0}=0$. Here we have used that

$$
\|f g\|_{H^{2}(\Omega)} \leq C\left(\|f\|_{B_{3,1}^{1}(\Omega)}\|g\|_{H^{2}(\Omega)}+\|f\|_{H^{2}(\Omega)}\|g\|_{B_{3,1}^{1}(\Omega)}\right)
$$

and have used (3.17). Hence there is some $0<T_{0} \leq 1$ such that

$$
\begin{aligned}
\left\|L(c) u-L\left(c_{0}\right) u\right\|_{Y_{T}} & \leq \frac{1}{4 C(1)}\|u\|_{X_{T}} \quad \text { for all } 0<T \leq T_{0},\|c\|_{X_{T}^{2}} \leq R \\
\left\|L\left(c_{1}\right) u-L\left(c_{2}\right) u\right\|_{Y_{T}} & \leq \frac{1}{4 C(1)}\|u\|_{X_{T}} \quad \text { for all } 0<T \leq T_{0},\left\|c_{j}\right\|_{X_{T}^{2}} \leq R, j=1,2,
\end{aligned}
$$

since $c_{0} \in \widetilde{X}_{T}^{2}$. This implies that $L(c): Y_{T} \rightarrow X_{T}$ is invertible and $\left\|L(c)^{-1}\right\|_{\mathcal{L}\left(Y_{T}, X_{T}\right)} \leq$ $\frac{4}{3} C(1) \leq 2 C(1)$ as well as

$$
\begin{aligned}
& \left\|L\left(c_{1}\right)^{-1}-L\left(c_{2}\right)^{-1}\right\|_{\mathcal{L}\left(Y_{T}, X_{T}\right)} \\
& \quad \leq 4 C(1)^{2}\left\|L\left(c_{1}\right)-L\left(c_{2}\right)\right\|_{\mathcal{L}\left(X_{T}, Y_{T}\right)} \leq C(R) T^{\frac{1}{8}}\left\|c_{1}-c_{2}\right\|_{X_{T}^{2}} .
\end{aligned}
$$

Moreover, we can choose $T_{0}$ so small that $\|c\|_{X_{T_{0}}^{2}} \leq R$ and $\left.c\right|_{t=0}=c_{0}$ implies $\| c-$ $c_{0} \|_{C^{0}\left(\overline{Q_{T}}\right)} \leq \varepsilon_{0}$ since $X_{T_{0}}^{2} \hookrightarrow C^{\frac{1}{8}}\left(\left[0, T_{0}\right] ; C^{0}(\bar{\Omega})\right)$, where $\varepsilon_{0}$ is as in Assumption 1.1] Then $|c(x, t)| \leq 1+\varepsilon_{0}$ for all $0 \leq t \leq T, x \in \bar{\Omega}$.

Hence we can write (3.7)-(3.10) as a fixed point equation

$$
u=S(c) L^{-1}(c) \mathcal{F}(u)=: \mathcal{G}(u),
$$


where $S(c): X_{T} \rightarrow X_{T}$ is defined by

$$
S(c)\left(\begin{array}{c}
\mathbf{v}^{\prime} \\
c^{\prime}
\end{array}\right)=\left(\begin{array}{c}
\mathbf{v}^{\prime} \\
\hat{\rho}(c)^{-1} c^{\prime}+\tilde{c}_{0}
\end{array}\right) \equiv S^{\prime}(c)+\left(\begin{array}{c}
0 \\
\tilde{c}_{0}
\end{array}\right) .
$$

In order to estimate $S(c)$, we use

$$
\left\|\left(\hat{\rho}\left(c_{1}\right)^{-1}-\hat{\rho}\left(c_{2}\right)^{-1}\right) c^{\prime}\right\|_{L^{2}\left(0, T ; H^{3}(\Omega)\right)} \leq C(R) T^{\frac{1}{4}}\left\|c_{1}-c_{2}\right\|_{X_{T}^{2}}\left\|c^{\prime}\right\|_{X_{T}^{2}}
$$

if $\left\|c_{j}\right\|_{X_{T}^{2}} \leq R$, which can be shown using that $X_{T}^{2} \hookrightarrow C^{\frac{1}{4}}\left([0, T] ; B_{2,1}^{3 / 2}(\Omega)\right)$ and $c_{1}-$ $\left.c_{2}\right|_{t=0}=\left.c^{\prime}\right|_{t=0}=0$. Moreover, we have

$$
\begin{aligned}
& \left\|\partial_{t}\left(\left(\hat{\rho}\left(c_{1}\right)^{-1}-\hat{\rho}\left(c_{2}\right)^{-1}\right) c^{\prime}\right)\right\|_{L^{2}\left(Q_{T}\right)} \\
& \quad \leq\left\|\left(\hat{\rho}\left(c_{1}\right)^{-1}-\hat{\rho}\left(c_{2}\right)^{-1}\right) \partial_{t} c^{\prime}\right\|_{L^{2}\left(Q_{T}\right)}+\|\left(\partial_{t}\left(\hat{\rho}\left(c_{1}\right)^{-1}-\hat{\rho}\left(c_{2}\right)^{-1}\right) c^{\prime} \|_{L^{2}\left(Q_{T}\right)}\right. \\
& \quad \leq C(R)\left(\left\|c_{1}-c_{2}\right\|_{B U C([0, T] ; C(\bar{\Omega}))}\left\|c^{\prime}\right\|_{X_{T}^{2}}+\left\|\partial_{t}\left(c_{1}-c_{2}\right)\right\|_{L^{2}\left(Q_{T}\right)}\left\|c^{\prime}\right\|_{B U C([0, T] ; C(\bar{\Omega}))}\right) \\
& \quad \leq C(R) T^{\frac{1}{4}}\left\|c_{1}-c_{2}\right\|_{X_{T}^{2}}\left\|c^{\prime}\right\|_{X_{T}^{2}},
\end{aligned}
$$

provided that $\left\|c_{j}\right\|_{X_{T}^{2}} \leq R$ and $c_{1}-\left.c_{2}\right|_{t=0}=\left.c^{\prime}\right|_{t=0}=0$. Here we have used that

$$
\|d\|_{B U C([0, T] ; C(\bar{\Omega}))} \leq C T^{\frac{1}{4}}\|d\|_{C^{\frac{1}{4}\left([0, T] ; B_{2,1}^{3 / 2}(\Omega)\right)}} \leq C^{\prime} T^{\frac{1}{4}}\|d\|_{X_{T}^{2}}
$$

for all $d \in X_{T}^{2}$ with $\left.d\right|_{t=0}=0$. Altogether this implies

$$
\left\|S\left(c_{1}\right)-S\left(c_{2}\right)\right\|_{\mathcal{L}\left(X_{T}^{0}, X_{T}\right)} \leq C(R) T^{\frac{1}{4}}\left\|c_{1}-c_{2}\right\|_{X_{T}^{2}}
$$

provided that $\left\|c_{j}\right\|_{X_{T}^{2}} \leq R$ and $c_{1}-\left.c_{2}\right|_{t=0}=0$, where $X_{T}^{0}=\left\{\left(, c^{\prime}\right) \in X_{T}:\left.c^{\prime}\right|_{t=0}=0\right\}$. Therefore we get

$$
\begin{aligned}
& \left\|S\left(c_{1}\right) L^{-1}\left(c_{1}\right)-S\left(c_{2}\right) L^{-1}\left(c_{2}\right)\right\|_{\mathcal{L}\left(Y_{T}^{0}, X_{T}\right)} \\
& \leq \quad\left\|S^{\prime}\left(c_{1}\right)\right\|_{\mathcal{L}\left(X_{T}\right)}\left\|L^{-1}\left(c_{1}\right)-L^{-1}\left(c_{2}\right)\right\|_{\mathcal{L}\left(Y_{T}, X_{T}\right)} \\
& \quad+\left\|S\left(c_{1}\right)-S\left(c_{2}\right)\right\|_{\mathcal{L}\left(X_{T}^{0}, X_{T}\right)}\left\|L^{-1}\left(c_{2}\right)\right\|_{\mathcal{L}\left(Y_{T}, X_{T}\right)} \\
& \quad \leq \quad C(R) T^{\frac{1}{4}}\left\|c_{1}-c_{2}\right\|_{X_{T}^{2}},
\end{aligned}
$$

where $Y_{T}^{0}=\left\{\left(\mathbf{f}, g, a, \mathbf{v}_{0}, c_{0}^{\prime}\right) \in Y_{T}: c_{0}^{\prime}=0\right\}$. - Note that $L(c)^{-1}\left(Y_{T}^{0}\right)=X_{T}^{0}$. - Because of Proposition 3.2, we have

$$
\begin{aligned}
& \left\|S\left(c_{1}\right) L^{-1}\left(c_{1}\right) \mathcal{F}\left(u_{1}\right)-S\left(c_{2}\right) L^{-1}\left(c_{2}\right) \mathcal{F}\left(u_{2}\right)\right\|_{X_{T}} \\
& \quad \leq\left\|S\left(c_{1}\right) L^{-1}\left(c_{1}\right)-S\left(c_{2}\right) L^{-1}\left(c_{2}\right)\right\|_{\mathcal{L}\left(Y_{T}^{0}, X_{T}\right)}\left\|\mathcal{F}\left(u_{1}\right)\right\|_{Y_{T}}+C\left\|\mathcal{F}\left(u_{1}\right)-\mathcal{F}\left(u_{2}\right)\right\|_{X_{T}} \\
& \quad \leq C(R) T^{\frac{1}{4}}\left\|u_{1}-u_{2}\right\|_{X_{T}}+C(T, R)\left\|u_{1}-u_{2}\right\|_{X_{T}} \leq \frac{1}{2}\left\|u_{1}-u_{2}\right\|_{X_{T}}
\end{aligned}
$$


for all sufficiently small $0<T \leq T_{0}$ and all $u_{j}=\left(\mathbf{v}_{j}, c_{j}\right) \in X_{T}$ with $\left\|u_{j}\right\|_{X_{T}} \leq R$ and $\left.c_{j}\right|_{t=0}=c_{0}$. Moreover,

$$
\begin{aligned}
& \left\|S(c) L^{-1}(c) \mathcal{F}(u)\right\|_{X_{T}} \\
& \quad \leq\left\|S(c) L^{-1}(c) \mathcal{F}(u)-S\left(\tilde{c}_{0}\right) L^{-1}\left(\tilde{c}_{0}\right) \mathcal{F}(0)\right\|_{X_{T}}+\left\|S\left(\tilde{c}_{0}\right) L^{-1}\left(\tilde{c}_{0}\right) \mathcal{F}(0)\right\|_{X_{T}} \\
& \quad \leq \frac{1}{2}\|u\|_{X_{T}}+\frac{R}{2} \leq R
\end{aligned}
$$

for all $\|u\|_{X_{T}} \leq R$. Hence by the contraction mapping principle there is a unique solution $u=(\mathbf{v}, c)$ of (3.7)-(3.10) with $\|u\|_{X_{T}} \leq R$.

Thus we have proved that for every $\mathbf{v}_{0} \in H_{n}^{1}(\Omega), c_{0} \in H^{2}(\Omega)$ there are some $T, R>0$ such that the system (3.7)-(3.10) has a unique solution $(\mathbf{v}, c) \in X_{T}$ with $\|(\mathbf{v}, c)\|_{X_{T}} \leq R$. In order to show that there is only one solution $(\mathbf{v}, c) \in X_{T}$ of (3.7)-(3.10), let $\left(\mathbf{v}^{\prime}, c^{\prime}\right) \in X_{T}$ be a second solution of (3.7)-(3.10) and let $R^{\prime}=$ $\max \left(R,\left\|\left(\mathbf{v}^{\prime}, c^{\prime}\right)\right\|_{X_{T}}\right)$. Then by the arguments above there is some $T^{\prime} \in(0, T]$ such that (3.7)-(3.10) has a unique solution $\left(\mathbf{v}^{\prime \prime}, c^{\prime \prime}\right) \in X_{T^{\prime}}$ (on the time interval $\left(0, T^{\prime}\right)$ ) with $\left\|\left(\mathbf{v}^{\prime \prime}, c^{\prime \prime}\right)\right\|_{X_{T^{\prime}}} \leq R^{\prime}$. Hence $\left.\left.(\mathbf{v}, c)\right|_{\left(0, T^{\prime}\right)} \equiv\left(\mathbf{v}^{\prime}, c^{\prime}\right)\right|_{\left(0, T^{\prime}\right)} \equiv\left(\mathbf{v}^{\prime \prime}, c^{\prime \prime}\right)$. Repeating this argument finitely many times (with a shift in time), we conclude that $(\mathbf{v}, c) \equiv\left(\mathbf{v}^{\prime}, c^{\prime}\right)$ on the full time interval $(0, T)$.

\section{$4 \quad$ Linearized System - Proof of Theorem 3.1}

In this section we will show unique solvability of the linear system

$$
\begin{aligned}
\partial_{t} \mathbf{v}-\operatorname{div} \widetilde{\mathbb{S}}\left(c_{0}, \mathbb{D} \mathbf{v}\right)+\frac{\varepsilon}{\beta \alpha} \nabla \operatorname{div}\left(\rho^{-4} \nabla c^{\prime}\right) & =\mathbf{f}_{1} & & \text { in } Q_{T}, \\
\partial_{t} c^{\prime}-\beta^{-1} \operatorname{div} \mathbf{v} & =f_{2} & & \text { in } Q_{T}, \\
\left(\mathbf{n} \cdot \widetilde{\mathbb{S}}\left(c_{0}, \mathbb{D}\left(P_{\sigma} \mathbf{v}\right)\right)\right)_{\tau}+\left.\tilde{\gamma}\left(c_{0}\right)\left(P_{\sigma} \mathbf{v}\right)_{\tau}\right|_{\partial \Omega} & =\mathbf{a} & & \text { on } S_{T}, \\
\left.\mathbf{n} \cdot \mathbf{v}\right|_{\partial \Omega}=\left.\partial_{n} c\right|_{\partial \Omega} & =0 & & \text { on } S_{T}, \\
\left.\left(\mathbf{v}, c^{\prime}\right)\right|_{t=0} & =\left(\mathbf{v}_{0}, c_{0}^{\prime}\right) & & \text { in } \Omega,
\end{aligned}
$$

where $\left(\mathbf{f}_{1}, f_{2}, \mathbf{a}, \mathbf{v}_{0}, c_{0}^{\prime}\right) \in Y_{T},\left(\mathbf{v}, c^{\prime}\right) \in X_{T}$, and $X_{T}, Y_{T}$ are as in Section 3 .

First of all, we can reduce to the case $\mathbf{a} \equiv 0$ by subtracting from $\mathbf{v}$ some $\mathbf{w} \in X_{T}^{1}$ such that $\left(\mathbf{n} \cdot \widetilde{\mathbb{S}}\left(c_{0}, \mathbb{D} \mathbf{w}\right)\right)_{\tau}+\left.\tilde{\gamma}\left(c_{0}\right) \mathbf{w}_{\tau}\right|_{\partial \Omega}=\mathbf{a}_{\tau},\left.\mathbf{w}\right|_{t=0}=0, \operatorname{div} \mathbf{w}=0,\left.\mathbf{w}\right|_{\partial \Omega}=0$. The existence of such a $\mathbf{w} \in X_{T}^{1}$, depending continuously on $\mathbf{a} \in H^{\frac{1}{4}, \frac{1}{2}}\left(S_{T}\right)^{d}$ with $\mathbf{a}_{n}=0$, follows directly from Lemma 2.3. For the following let

$$
T_{\gamma} \mathbf{u}=\left(\mathbf{n} \cdot \widetilde{\mathbb{S}}\left(c_{0}, \mathbb{D} \mathbf{u}\right)\right)_{\tau}+\left.\tilde{\gamma}\left(c_{0}\right) \mathbf{u}_{\tau}\right|_{\partial \Omega} \quad \text { for all } \mathbf{u} \in H^{2}(\Omega)^{d}
$$

Now we will reformulate the system above in an appropriate way assuming that $\left(\mathbf{v}, c^{\prime}\right) \in X_{T}$. Since (4.2) depends only on div $\mathbf{v}$, we will use the Helmholtz decomposition to decompose $\mathbf{v}$. More precisely, using $L^{2}(\Omega)^{d}=L_{\sigma}^{2}(\Omega) \oplus G_{2}(\Omega)$ and applying 
$P_{\sigma}$ and $\left(I-P_{\sigma}\right)$ to (4.1), we obtain that (4.1) is equivalent to

$$
\begin{aligned}
\partial_{t} \mathbf{w}-P_{\sigma} \operatorname{div} \widetilde{\mathbb{S}}\left(c_{0}, \mathbb{D} \mathbf{w}\right)-P_{\sigma} \operatorname{div} \widetilde{\mathbb{S}}\left(c_{0}, \nabla^{2} G(\operatorname{div} \mathbf{v})\right) & =P_{\sigma} \mathbf{f}_{1}, \\
\partial_{t} \nabla G(\operatorname{div} \mathbf{v})-\left(I-P_{\sigma}\right) \operatorname{div} \widetilde{\mathbb{S}}\left(c_{0}, \mathbb{D} \mathbf{v}\right)+\frac{\varepsilon}{\alpha \beta} \nabla \operatorname{div}\left(\rho_{0}^{-4} \nabla c^{\prime}\right) & =\left(I-P_{\sigma}\right) \mathbf{f}_{1},
\end{aligned}
$$

where $\mathbf{w}=P_{\sigma} \mathbf{v}$ and $\mathbf{v}=\mathbf{w}+\nabla G(\operatorname{div} \mathbf{v})$ and $G$ is defined by (3.4)-(3.5). In the following let $g=\operatorname{div} \mathbf{v}$.

In order to determine the principal part of (4.6), we use that

$$
\begin{aligned}
& P_{\sigma} \operatorname{div} \widetilde{\mathbb{S}}\left(c_{0}, \nabla^{2} G(\operatorname{div} \mathbf{v})\right)=P_{\sigma} \operatorname{div}\left(2 \tilde{\nu}\left(c_{0}\right) \nabla^{2} G(\operatorname{div} \mathbf{v})\right)+P_{\sigma} \nabla\left(\tilde{\eta}\left(c_{0}\right) \operatorname{div} \mathbf{v}\right) \\
& \quad=P_{\sigma} \nabla \operatorname{div}\left(\left(2 \tilde{\nu}\left(c_{0}\right)\right) \nabla G(\operatorname{div} \mathbf{v})\right)-P_{\sigma} \operatorname{div}\left(2 \nabla \tilde{\nu}\left(c_{0}\right) \otimes \nabla G(\operatorname{div} \mathbf{v})\right) \\
& \quad=-P_{\sigma} \operatorname{div}\left(2 \nabla \tilde{\nu}\left(c_{0}\right) \otimes \nabla G(\operatorname{div} \mathbf{v})\right) \equiv B_{1} g
\end{aligned}
$$

Moreover, testing (4.7) with $\nabla \varphi$, where $\varphi \in C_{0}^{\infty}\left(0, T ; H_{(0)}^{1}(\Omega)\right)$, one sees that (4.7) is equivalent to

$$
\begin{aligned}
&-\left\langle\partial_{t} g, \varphi\right\rangle_{L^{2}\left(0, T ; H_{(0)}^{-1}\right)}-\left(\operatorname{div} \widetilde{\mathbb{S}}\left(c_{0}, \mathbb{D} \mathbf{v}\right), \nabla \varphi\right)_{Q_{T}} \\
&+\frac{\varepsilon}{\alpha \beta}\left(\nabla \operatorname{div}\left(\rho_{0}^{-4} \nabla \tilde{c}\right), \nabla \varphi\right)_{Q_{T}}=\left(\left(I-P_{\sigma}\right) \mathbf{f}_{1}, \nabla \varphi\right)_{Q_{T}}
\end{aligned}
$$

for all $\varphi \in C_{0}^{\infty}\left(0, T ; H_{(0)}^{1}(\Omega)\right)$, where we have used again the orthogonal decomposition $L^{2}(\Omega)^{d}=L_{\sigma}^{2}(\Omega) \oplus G_{2}(\Omega)$ and the fact that $g=\operatorname{div}_{n} \mathbf{v} \in H^{1}\left(0, T ; H_{(0)}^{-1}(\Omega)\right)$ if $\mathbf{v} \in X_{T}^{1}$. Moreover, we note that

$$
\begin{aligned}
& \operatorname{div} \widetilde{\mathbb{S}}\left(c_{0}, \nabla^{2} G(\operatorname{div} \mathbf{v})\right)=\operatorname{div}\left(2 \tilde{\nu}\left(c_{0}\right) \nabla^{2} G(\operatorname{div} \mathbf{v})\right)+\nabla\left(\tilde{\eta}\left(c_{0}\right) g\right) \\
& \quad=2 \tilde{\nu}\left(c_{0}\right) \nabla g+\nabla\left(\tilde{\eta}\left(c_{0}\right) g\right)+2\left(\nabla \tilde{\nu}\left(c_{0}\right)\right) \cdot \nabla^{2} G(\operatorname{div} \mathbf{v}) \\
& \quad=\nabla\left(\left(2 \tilde{\nu}\left(c_{0}\right)+\tilde{\eta}\left(c_{0}\right)\right) g\right)+2\left(\nabla \tilde{\nu}\left(c_{0}\right)\right) \cdot \nabla^{2} G(\operatorname{div} \mathbf{v})-\left(2 \nabla \tilde{\nu}\left(c_{0}\right)\right) g .
\end{aligned}
$$

Hence

$$
\left(\operatorname{div} \widetilde{\mathbb{S}}\left(c_{0}, \mathbb{D} \nabla G(g)\right), \nabla \varphi\right)=-\left\langle\Delta_{N}\left(a\left(c_{0}\right) g\right), \varphi\right\rangle_{H_{(0)}^{-1}, H_{(0)}^{1}}+\left\langle B_{2} g, \varphi\right\rangle_{H_{(0)}^{-1}, H_{(0)}^{1}},
$$

where $a\left(c_{0}\right)=2 \tilde{\nu}\left(c_{0}\right)+\tilde{\eta}\left(c_{0}\right)$ and $B_{2}$ is defined by the equation.

Therefore we can reformulate (4.2)-(4.7) with $\mathbf{a} \equiv 0$ more abstractly as

$$
\begin{aligned}
\partial_{t} u+\mathcal{A} u+\mathcal{B} u & =\left(\begin{array}{c}
f_{2} \\
\operatorname{div}_{n}\left(I-P_{\sigma}\right) \mathbf{f}_{1} \\
P_{\sigma} \mathbf{f}_{1}
\end{array}\right)=: f \\
\left.u\right|_{t=0} & =\left(\begin{array}{c}
c_{0}^{\prime} \\
g_{0} \\
\mathbf{w}_{0}
\end{array}\right)=: u_{0}
\end{aligned}
$$


where $u=\left(c^{\prime}, g, \mathbf{w}\right)^{T}, g_{0}=\operatorname{div} \mathbf{v}_{0}, \mathbf{v}_{0}=\mathbf{w}_{0}+\nabla G\left(g_{0}\right)$, and

$$
\begin{aligned}
\mathcal{A} u & =\left(\begin{array}{cc}
A_{1} & A_{2} \\
0 & -P_{\sigma} \operatorname{div} \widetilde{\mathbb{S}}\left(c_{0}, \mathbb{D} \mathbf{w}\right)
\end{array}\right)\left(\begin{array}{c}
\left(c^{\prime}, g\right)^{T} \\
\mathbf{w}
\end{array}\right), \\
A_{1} & =\left(\begin{array}{cc}
0 & -\beta^{-1} P_{0} \\
\frac{\varepsilon}{\alpha \beta} \Delta_{N}\left(\operatorname{div} \rho_{0}^{-4} \nabla \cdot\right) & -\Delta_{N}\left(a\left(c_{0}\right) \cdot\right)
\end{array}\right), \\
A_{2} \mathbf{w} & =\left(\begin{array}{c}
0 \\
-\operatorname{div}_{n}\left(\operatorname{div} \widetilde{\mathbb{S}}\left(c_{0}, \mathbb{D} \mathbf{w}\right)\right)
\end{array}\right), \mathcal{B} u=\left(\begin{array}{c}
0 \\
B_{2} g \\
-B_{1} g
\end{array}\right) .
\end{aligned}
$$

and the domains of $\mathcal{A}, \mathcal{B}$ are defined as

$$
\begin{aligned}
\mathcal{D}(\mathcal{A}) & =\mathcal{D}(\mathcal{B})=\left\{\left(c^{\prime}, g, \mathbf{w}\right)^{T}:\left(c^{\prime}, g\right) \in \mathcal{D}\left(A_{1}\right), \mathbf{w} \in H^{2}(\Omega)^{d} \cap L_{\sigma}^{2}(\Omega): T_{\gamma} \mathbf{w}=0\right\} \\
\mathcal{D}\left(A_{1}\right) & =\left(H^{3}(\Omega) \cap H_{N}^{2}(\Omega)\right) \times H_{(0)}^{1}(\Omega) .
\end{aligned}
$$

We consider $\mathcal{A}$ and $\mathcal{B}$ as unbounded operators on

$$
H=H_{1} \times L_{\sigma}^{2}(\Omega) \quad \text { where } H_{1}=H^{1}(\Omega) \times H_{(0)}^{-1}(\Omega) .
$$

For the following analysis it will be crucial that $B_{1}$ and $B_{2}$ are of lower order compared to $\mathcal{A}$. More precisely, we have:

Lemma 4.1 Let $s \in\left(\frac{1}{2}, 1\right]$ and $c_{0} \in H^{2}(\Omega)$. Then there are constants $C\left(c_{0}\right)$, $C^{\prime}\left(c_{0}, s\right)>0$ such that

$$
\begin{aligned}
\left\|B_{1} g\right\|_{L^{2}(\Omega)} & \leq C^{\prime}\left(s, c_{0}\right)\|g\|_{H^{s}(\Omega)}, \\
\left\|B_{2} g\right\|_{H_{(0)}^{-1}(\Omega)} & \leq C\left(c_{0}\right)\|g\|_{H^{\frac{1}{2}}(\Omega)}
\end{aligned}
$$

for all $g \in H_{(0)}^{1}(\Omega)$, where $B_{1}, B_{2}$ are as above.

Proof: By the definition of $B_{1}$ and (4.8), we have

$$
\begin{aligned}
\left\|B_{1} g\right\|_{L^{2}(\Omega)} & =\left\|P_{\sigma} \operatorname{div} \widetilde{\mathbb{S}}\left(c_{0}, \mathbb{D} \nabla G(g)\right)\right\|_{L^{2}(\Omega)} \\
& \leq C\|\nabla \tilde{\nu}\|_{H^{1}(\Omega)}\|\nabla G(g)\|_{H^{1+s}(\Omega)} \leq C\left(s, c_{0}\right)\|g\|_{H^{s}(\Omega)}
\end{aligned}
$$

for every $s>\frac{1}{2}$ since $c_{0} \in H^{2}(\Omega), \Delta_{N}^{-1}: H^{s}(\Omega) \rightarrow H^{s+2}(\Omega)$ for all $s \in[0,1]$ due to (2.9), and $\|f g\|_{H^{1}} \leq C_{s}\|f\|_{H^{s}}\|g\|_{H^{1}}$ if $s>\frac{d}{2}$.

Finally, $B_{2}$ satisfies

$$
\begin{aligned}
\left\|B_{2} g\right\|_{H_{(0)}^{-1}(\Omega)} & \leq 2\left\|\left(\nabla \tilde{\nu}\left(c_{0}\right)\right) \cdot \nabla^{2} G(g)-\nabla \tilde{\nu}\left(c_{0}\right) g\right\|_{L^{2}(\Omega)} \\
& \leq C^{\prime}\left\|\nabla c_{0}\right\|_{L^{6}(\Omega)}\|g\|_{L^{3}(\Omega)} \leq C\left(c_{0}\right)\|g\|_{H^{\frac{1}{2}}(\Omega)}
\end{aligned}
$$

due to (2.9).

Because of the triangle structure of $\mathcal{A}$, it is sufficient to prove that $-A_{1}$ and $P_{\sigma}\left(\operatorname{div} \widetilde{\mathbb{S}}\left(c_{0}, \cdot\right)\right)$ generate analytic semigroups in order to have the same for $-\mathcal{A}$. 
Lemma 4.2 Let $A_{1}$ and $H_{1}$ be as above. Then $-A_{1}$ generates a bounded analytic semigroup. Moreover, $\left\|A_{1}\left(c^{\prime}, g\right)^{T}\right\|_{H_{1}}+\left|m\left(c^{\prime}\right)\right|$ is equivalent to $\|g\|_{H^{1}}+\left\|c^{\prime}\right\|_{H^{3}}$.

Proof: Let $H_{0}=H_{(0)}^{1}(\Omega) \times H_{(0)}^{-1}(\Omega)$. Then $A_{1}$ leaves $H_{0}$ invariant. We first show that $-\left.A_{1}\right|_{H_{0}}$ generates an analytic semigroup. To this end we use that

$$
-\left.A_{1}\right|_{H_{0}}=\left(\begin{array}{cc}
0 & \beta^{-1} I \\
-A & -B
\end{array}\right), \quad \text { where } A=\frac{\varepsilon}{\alpha \beta} \Delta_{N} \operatorname{div}\left(\rho_{0}^{-4} \nabla \cdot\right), B=-\Delta_{N}\left(a\left(c_{0}\right) \cdot\right) .
$$

Here $\mathcal{D}(A)=H^{3}(\Omega) \cap H_{N}^{2}(\Omega) \cap H_{(0)}^{1}(\Omega), \mathcal{D}(B)=H_{(0)}^{1}(\Omega)$. Without loss of generality let $\beta=1$. (Otherwise replace $A_{1}$ by $\beta A_{1}$.) Because of [12, Theorem 1.1], $-\left.A_{1}\right|_{H_{0}}$ generates an analytic semigroup on $H_{0}$ provided that the following conditions are satisfied:

H1 $A, B$ are positive self-adjoint operators on $H_{(0)}^{-1}(\Omega)$ with dense domains $\mathcal{D}(A)$, $\mathcal{D}(B)$. A has a compact resolvent.

H2 $\mathcal{D}\left(A^{\frac{1}{2}}\right)=\mathcal{D}(B)$ and there are constants $0<\rho_{1}<\rho_{2}<\infty$ such that $\rho_{1} A^{\frac{1}{2}} \leq$ $B \leq \rho_{2} A^{\frac{1}{2}}$.

We note that, if these conditions are satisfied, then $e^{-\left.A_{1}\right|_{H_{0}} t}$ is an exponentially decreasing semigroup of contractions on $H_{0}$ equipped with the norm of $\mathcal{D}\left(A^{\frac{1}{2}}\right) \times$ $H_{(0)}^{-1}(\Omega)$. In particular, $A_{1}$ is invertible.

Let us verify the conditions above. First of all, $A, B$ are positive and symmetric since

$$
\begin{aligned}
(A u, v)_{H_{(0)}^{-1}} & =-\frac{\alpha \beta}{\varepsilon}\left(\operatorname{div}\left(\rho_{0}^{-4} \nabla u\right), v\right)_{L^{2}(\Omega)}=\frac{\alpha \beta}{\varepsilon}\left(\rho_{0}^{-4} \nabla u, \nabla v\right)_{L^{2}(\Omega)}=(u, A v)_{H_{(0)}^{-1}} \\
\left(B u^{\prime}, v^{\prime}\right)_{H_{(0)}^{-1}} & =\left(a\left(c_{0}\right) u^{\prime}, v^{\prime}\right)_{L^{2}(\Omega)}=\left(u^{\prime}, B v^{\prime}\right)_{H_{(0)}^{-1}}
\end{aligned}
$$

for all $u, v \in \mathcal{D}(A), u^{\prime}, v^{\prime} \in \mathcal{D}(B)$, where we have used (2.6). Moreover, with the aid of the Lemma of Lax-Milgram and standard elliptic regularity theory one easily shows that $A$ and $B$ are invertible. Hence $A, B$ are self-adjoint. In order to verify H2, we use that there are constants $c_{0}, C_{0}>0$ such that

$$
c_{0}(\nabla u, \nabla u)_{L^{2}(\Omega)} \leq(A u, u)_{H_{(0)}^{-1}}=\frac{\alpha \beta}{\varepsilon}\left(\rho_{0}^{-4} \nabla u, \nabla u\right)_{L^{2}(\Omega)} \leq C_{0}(\nabla u, \nabla u)_{L^{2}(\Omega)}
$$

since $\rho_{0}^{-4}$ is bounded above and below, where

$$
(\nabla u, \nabla u)_{L^{2}}=-\left(\Delta_{N} u, u\right)_{L^{2}}=\left(\left(-\Delta_{N}\right)^{2} u, u\right)_{H_{(0)}^{-1}}=\left\|\Delta_{N} u\right\|_{H_{(0)}^{-1}}^{2}
$$

Hence $c_{0}\left(-\Delta_{N}\right)^{2} \leq A \leq C_{0}\left(-\Delta_{N}\right)^{2}$ in $H_{(0)}^{-1}(\Omega)$. This implies that there are $c_{1}, c_{2}>0$

$$
\begin{aligned}
c_{1}\left(\left(-\Delta_{N}\right) u, u\right)_{H_{(0)}^{-1}} & =c_{1}\left\|\left(-\Delta_{N}\right)^{\frac{1}{2}} u\right\|_{H_{(0)}^{-1}(\Omega)}^{2} \leq\left\|A^{\frac{1}{4}} u\right\|_{H_{(0)}^{-1}}=\left(A^{\frac{1}{2}} u, u\right)_{H_{(0)}^{-1}} \\
& \leq c_{2}\left\|\left(-\Delta_{N}\right)^{\frac{1}{2}} u\right\|_{H_{(0)}^{-1}(\Omega)}^{2}=c_{2}\left(\left(-\Delta_{N}\right) u, u\right)_{H_{(0)}^{-1}}
\end{aligned}
$$


because of [23, Chapter I, Corollary 7.1]. Thus $c_{1}\left(-\Delta_{N}\right) \leq A^{\frac{1}{2}} \leq c_{2}\left(-\Delta_{N}\right)$. [23, Chapter I, Corollary 7.1] also implies $\mathcal{D}\left(A^{\frac{1}{2}}\right)=H_{(0)}^{1}(\Omega)=\mathcal{D}\left(-\Delta_{N}\right)$. Moreover, we have that there are $c_{3}, c_{4}>0$ such that

$c_{3}\left(-\Delta_{N} u, u\right)_{H_{(0)}^{-1}}=c_{3}(u, u)_{L^{2}} \leq\left(a\left(c_{0}\right)^{-1} u, u\right)_{L^{2}}=(B u, u)_{H_{(0)}^{-1}} \leq c_{4}\left(-\Delta_{N} u, u\right)_{H_{(0)}^{-1}}$

for all $u \in H_{(0)}^{1}(\Omega)$. Combining this with the previous estimates, we obtain $\rho_{1} A^{\frac{1}{2}} \leq$ $B \leq \rho_{2} A^{\frac{1}{2}}$ for some $0<\rho_{1} \leq \rho_{2}<\infty$.

Hence we have proved H1-H2 and conclude that $-\left.A_{1}\right|_{H_{0}}$ generates a bounded analytic semigroup on $H_{0}$. Moreover, $H_{1}=H_{0} \oplus\{(0, m): m \in \mathbb{R}\}$ and $A_{1}\left(I-P_{0}\right)=$ $\left(I-P_{0}\right) A_{1}=0$. Therefore there is some $\delta \in\left(\frac{\pi}{2}, \pi\right)$ such that $\lambda+A_{1}: \mathcal{D}\left(A_{1}\right) \rightarrow H_{1}$ is invertible for all $\lambda \in \Sigma_{\delta}$. Moreover, the resolvent estimate $\left\|\left(\lambda+A_{1}\right)^{-1}\right\|_{\mathcal{L}(H)} \leq \frac{C}{|\lambda|}$ for all $\lambda \in \Sigma_{\delta}$ follows from the corresponding estimate for $\left.A_{1}\right|_{H_{0}}$. Therefore $-A_{1}$ generates a bounded analytic semigroup on $H_{1}$.

The equivalence of norms follows from the invertibility of $\left.A_{1}\right|_{H_{0}}$ and the boundedness of $A_{1}:\left(H^{3}(\Omega) \cap H_{N}^{2}(\Omega)\right) \times H_{(0)}^{1}(\Omega) \rightarrow H_{1}$.

Remark 4.3 As shown above there is some $\delta>\frac{\pi}{2}$ such that $\Sigma_{\delta} \subseteq \rho\left(-A_{1}\right)$. In the special case that $\tilde{\nu} \equiv \nu_{0}, \tilde{\eta} \equiv \eta_{0}$, and $\rho_{0} \equiv 1$ are constant, we have $a\left(c_{0}\right) \equiv 2 \nu_{0}+\eta_{0}$ and $\sigma\left(-A_{1}\right)$ consists of the eigenvalues

$$
\lambda_{n}^{ \pm}=\sqrt{\frac{\varepsilon}{\alpha \beta} \mu_{n}} e^{ \pm i \theta}, \quad e^{i \theta}=-\kappa+i \sqrt{1-\kappa^{2}}, \quad \kappa=\frac{\alpha \beta^{2}\left(2 \nu_{0}+\mu_{0}\right)}{\varepsilon}
$$

provided that $0<\kappa<1$, where $\mu_{n}$ are the eigenvalues of $\Delta_{N} \Delta$, cf. [12, Lemma A.1]. Note that $\frac{\pi}{2}<\theta<\pi$ and $\theta \rightarrow \frac{\pi}{2}$ as $\kappa \rightarrow 0$. Hence $\delta>\frac{\pi}{2}$ above can be arbitrarily close to $\frac{\pi}{2}$ in certain situations.

Because of the triangular structure of $A$, we conclude from the latter lemma:

Proposition $4.4-\mathcal{A}$ generates a bounded analytic semigroup on $H$. Moreover, $\|\mathcal{A} u\|_{H}+\|u\|_{H}$ is equivalent to $\|\mathbf{w}\|_{H^{2}}+\|g\|_{H^{1}}+\|c\|_{H^{3}}$, where $u=\left(c^{\prime}, g, \mathbf{w}\right)^{T}$.

Proof: Let $A_{\gamma} \mathbf{v} \equiv A_{\gamma}\left(c_{0}\right) \mathbf{v}=-P_{\sigma} \operatorname{div} \widetilde{\mathbb{S}}\left(c_{0}, \mathbb{D} \mathbf{v}\right)=-P_{\sigma} \operatorname{div}\left(\tilde{\nu}\left(c_{0}\right) \mathbb{D} \mathbf{v}\right)$ for all $\mathbf{v} \in$ $\mathcal{D}\left(A_{\gamma}\right)=\left\{\mathbf{u} \in H^{2}(\Omega)^{d} \cap L_{\sigma}^{2}(\Omega): T_{\gamma} \mathbf{u}=0\right\}$. Then $-A_{\gamma}\left(c_{0}\right): \mathcal{D}\left(A_{\gamma}\right) \subset L_{\sigma}^{2}(\Omega) \rightarrow$ $L_{\sigma}^{2}(\Omega)$ generates a bounded analytic $C^{0}$-semigroup because of Theorem A.1 below. Moreover, $A_{\gamma}$ is invertible and $\|A \mathbf{v}\|_{L_{\sigma}^{2}(\Omega)}$ is equivalent to $\|\mathbf{v}\|_{H^{2}(\Omega)}$ because of [4, Lemma 4]. Hence there is some $\delta>\frac{\pi}{2}$ such that $(\lambda+A)^{-1}$ exists for all $\lambda \in \Sigma_{\delta}$ and

$$
(\lambda+A)^{-1}=\left(\begin{array}{cc}
\left(\lambda+A_{1}\right)^{-1} & -\left(\lambda+A_{1}\right)^{-1} A_{2}\left(\lambda+A_{\gamma}\right)^{-1} \\
0 & \left(\lambda+A_{\gamma}\right)^{-1}
\end{array}\right) .
$$

Using

$$
|\lambda|\left\|\left(\lambda+A_{\gamma}\right)^{-1}\right\|_{\mathcal{L}\left(L_{\sigma}^{2}\right)}+\left\|A_{\gamma}\left(\lambda+A_{\gamma}\right)^{-1}\right\|_{\mathcal{L}\left(L_{\sigma}^{2}\right)} \leq C_{\delta} \quad \text { for all } \lambda \in \Sigma_{\delta}
$$


we conclude that

$$
\begin{aligned}
\left\|A_{2}\left(\lambda+A_{\gamma}\right)^{-1} \mathbf{f}_{2}\right\|_{H_{1}} & \leq C\left\|\left(\lambda+A_{\gamma}\right)^{-1} \mathbf{f}_{2}\right\|_{H^{2}(\Omega)} \\
& \leq C^{\prime}\left\|A_{\gamma}\left(\lambda+A_{\gamma}\right)^{-1} \mathbf{f}_{2}\right\|_{L_{\sigma}^{2}(\Omega)} \leq C^{\prime \prime}\left\|\mathbf{f}_{2}\right\|_{L^{2}(\Omega)}
\end{aligned}
$$

for all $\mathbf{f}_{2} \in L_{\sigma}^{2}(\Omega), \lambda \in \Sigma_{\delta}$. Therefore we easily obtain

$$
\left\|(\lambda+\mathcal{A})^{-1}\right\|_{H} \leq \frac{C}{|\lambda|} \quad \text { uniformly in } \lambda \in \Sigma_{\delta} .
$$

Hence $-\mathcal{A}$ generates a bounded analytic $C^{0}$-semigroup. Finally, the equivalence of norms can be easily shown using the resolvent identity above for $\lambda=1$ and the corresponding statement in Lemma 4.2.

Corollary 4.5 Let $c_{0} \in H^{2}(\Omega)$. Then $\mathcal{A}+\mathcal{B}$ generates an analytic $C^{0}$-semigroup. Moreover, $\|(\mathcal{A}+\mathcal{B}) u\|_{H}+\|u\|_{H}$ is equivalent to $\|\mathbf{w}\|_{H^{2}}+\|g\|_{H^{1}}+\|c\|_{H^{3}}$, where $u=(c, g, \mathbf{w})^{T} \in \mathcal{D}(\mathcal{A})$.

Proof: The corollary follows from the fact that for every $\varepsilon>0$ there is a constant $C_{\varepsilon}>0$ such that

$$
\|\mathcal{B} u\|_{H} \leq \varepsilon\|\mathcal{A} u\|_{H}+C_{\varepsilon}\|u\|_{H}
$$

for all $u \in \mathcal{D}(A)$ and a standard result from semigroup theory, cf. e.g. [30, Chapter 3 , Theorem 2.1]. The latter estimate follows from

$$
\begin{aligned}
\|\mathcal{B} u\|_{H} & \leq C\left(c_{0}\right)\|g\|_{H^{\frac{3}{4}(\Omega)}} \leq C\left(c_{0}\right)\|g\|_{L^{2}(\Omega)}^{\frac{1}{4}}\|g\|_{H^{1}(\Omega)}^{\frac{3}{4}} \leq C\left(c_{0}\right)\|g\|_{H_{(0)}^{-1}(\Omega)}^{\frac{1}{8}}\|g\|_{H^{1}(\Omega)}^{\frac{7}{8}} \\
& \leq C\left(c_{0}\right)\|\mathcal{A} u\|_{H}^{\frac{7}{8}}\|u\|_{H}^{\frac{1}{8}} \leq \varepsilon\|\mathcal{A} u\|_{H}+C_{\varepsilon}\left(c_{0}\right)\|u\|_{H}
\end{aligned}
$$

for every $\varepsilon>0$, where $u=(c, g, \mathbf{w})$ and we have used Lemma 4.1, $H^{\frac{3}{4}}(\Omega)=$ $\left(L^{2}(\Omega), H^{1}(\Omega)\right)_{\frac{3}{4}, 2}$, and (2.7).

Lemma 4.6 Let $\mathcal{A}, \mathcal{D}(\mathcal{A}), H$ be defined as above. Then

$$
(\mathcal{D}(\mathcal{A}), H)_{\frac{1}{2}, 2}=H_{N}^{2}(\Omega) \times L_{(0)}^{2}(\Omega) \times\left(H^{1}(\Omega)^{d} \cap L_{\sigma}^{2}(\Omega)\right) .
$$

Proof: We only need to show that

$$
\begin{aligned}
\left(H_{(0)}^{1}(\Omega), H_{(0)}^{-1}(\Omega)\right)_{\frac{1}{2}, 2} & =L_{(0)}^{2}(\Omega) \\
\left(H^{3}(\Omega) \cap H_{N}^{2}(\Omega), H^{1}(\Omega)\right)_{\frac{1}{2}, 2} & =H_{N}^{2}(\Omega) \\
\left(\mathcal{D}\left(A_{\gamma}\right), L_{\sigma}^{2}(\Omega)\right)_{\frac{1}{2}, 2} & =H^{1}(\Omega)^{d} \cap L_{\sigma}^{2}(\Omega),
\end{aligned}
$$

where $\mathcal{D}\left(A_{\gamma}\right)=\left\{\mathbf{u} \in H^{2}(\Omega)^{d} \cap L_{\sigma}^{2}(\Omega): T_{\gamma} \mathbf{u}=0\right\}$. The first equality follows from $H_{(0)}^{1}(\Omega)=P_{0} H^{1}(\Omega), L_{(0)}^{2}(\Omega)=P_{0} L^{2}(\Omega), H_{(0)}^{-1}(\Omega)=P_{0} H^{-1}(\Omega)$ and [35, Section 1.2.4, Theorem]. The second identity is proved using that $H^{3}(\Omega) \cap H_{N}^{2}(\Omega)=$ 
$\left(1-\Delta_{N}\right)^{-1} H^{1}(\Omega), H^{1}(\Omega)=\left(1-\Delta_{N}\right)^{-1} H^{-1}(\Omega)$, and $H_{N}^{2}(\Omega)=\left(1-\Delta_{N}\right)^{-1} L^{2}(\Omega)$. The third identity follows from Lemma A.2, below.

Proof of Theorem 3.1: As seen at the beginning of Section 4 we can assume without loss of generality that $\mathbf{a} \equiv 0$. Let $\left(\mathbf{f}_{1}, f_{2}, 0, \mathbf{v}_{0}, c_{0}^{\prime}\right) \in Y_{T}$ and $f \in L^{2}(0, T ; H)$ be defined as in (4.9) and extend $f$ by zero for $t \geq T$. Moreover, let $g_{0}=\operatorname{div} \mathbf{v}_{0}$, $\mathbf{w}_{0}=P_{\sigma} \mathbf{v}_{0}$, and let $u_{0}$ be as in (4.10).

Applying Theorem 2.1 there is a unique solution $u$ of

$$
\begin{aligned}
\frac{d u}{d t}(t)+\mathcal{A} u(t)+\mathcal{B} u(t) & =f(t), \quad t>0, \\
u(0) & =u_{0}
\end{aligned}
$$

and

$$
\left\|\left(\partial_{t} u,(\mathcal{A}+\mathcal{B}) u\right)\right\|_{L^{2}(0, \infty ; H)} \leq C\left(\|f\|_{L^{2}(0, \infty ; H)}+\left\|u_{0}\right\|_{(\mathcal{D}(\mathcal{A}), H)_{\frac{1}{2}, 2}}\right)
$$

Using that $\|u\|_{L^{2}\left(0, T_{0} ; H\right)} \leq C\left(T_{0}\right)\left(\left\|\partial_{t} u\right\|_{L^{2}\left(0, T_{0} ; H\right)}+\left\|u_{0}\right\|_{H}\right)$ for any fixed $0<T_{0}<\infty$, we obtain that $u$ restricted to $(0, T)$ satisfies

$$
\left\|\left(u, \partial_{t} u,(\mathcal{A}+\mathcal{B}) u\right)\right\|_{L^{2}(0, T ; H)} \leq C\left(T_{0}\right)\left(\|f\|_{L^{2}(0, T ; H)}+\left\|u_{0}\right\|_{(\mathcal{D}(\mathcal{A}), H)_{\frac{1}{2}, 2}}\right)
$$

uniformly in $0<T \leq T_{0}$ and $\left(f, u_{0}\right)$. Hence $u=\left(c^{\prime}, g, \mathbf{w}\right)$ solves (4.2)-(4.7) with $G(\operatorname{div} \mathbf{v})=\Delta_{N}^{-1} g$. Therefore $\left(\mathbf{v}, c^{\prime}\right)$ with $\mathbf{v}=\mathbf{w}+\nabla \Delta_{N}^{-1} g$ solves (4.1)-(4.5), which implies

$$
L\left(c_{0}\right)\left(\begin{array}{l}
\mathbf{v} \\
c^{\prime}
\end{array}\right)=\left(\begin{array}{c}
\mathbf{f}_{1} \\
0 \\
f_{2}
\end{array}\right) .
$$

The estimate of $\left(\mathbf{v}, c^{\prime}\right) \in X_{T}$, the continuity of $L\left(c_{0}\right)^{-1}: Y_{T} \rightarrow X_{T}$ follows from the estimate above and the equivalence of norms stated in Corollary 4.5 .

\section{A Stokes Operator with Navier Boundary Condi- tions}

In this appendix we summarize some results for the Stokes operator with variable viscosity in the case of Navier boundary conditions. More detailed information can be found in [1, Chapter 5].

We consider

$$
A_{\gamma}(c): \mathcal{D}\left(A_{\gamma}(c)\right) \subset L_{\sigma}^{2}(\Omega) \rightarrow L_{\sigma}^{2}(\Omega): \mathbf{v} \mapsto A_{\gamma}(c) \mathbf{v}:=-P_{2} \operatorname{div}(2 \nu(c) D \mathbf{v}),
$$

where $c \in W_{q}^{1}(\Omega), q>d$, and

$$
\mathcal{D}\left(A_{\gamma}(c)\right)=\left\{\mathbf{u} \in H^{2}(\Omega)^{d} \cap L_{\sigma}^{2}(\Omega):(2 \mathbf{n} \cdot \nu(c) \mathbb{D} \mathbf{u})_{\tau}+\gamma(c) \mathbf{u}_{\tau}=0\right\} .
$$


Here we assume that $c \in W_{q}^{1}(\Omega)$ for some $q>d, \gamma \in C^{1}(\mathbb{R})$ with $0 \leq \gamma(s)<\infty$ for all $s \in \mathbb{R}$, and $\Omega \subset \mathbb{R}^{d}, d=2,3$, is a bounded domains with $C^{3}$-boundary. - We note that $H^{2}(\Omega) \hookrightarrow W_{q}^{1}(\Omega)$ for some $q>d$ if $d=2,3$. - If $\inf _{s \in \mathbb{R}} \gamma(s)=0$ we assume additionally that $\Omega$ does not have an axis of symmetry, i.e., $R=\{0\}$, where

$$
\begin{array}{ll}
R=\left\{\mathbf{v} \in H_{n}^{1}(\Omega): \mathbf{v}(x)=\mathbf{a}+\mathbf{b} \wedge x, \mathbf{a}, \mathbf{b} \in \mathbb{R}^{3}\right\} & \text { if } d=3, \\
R=\left\{\mathbf{v} \in H_{n}^{1}(\Omega): \mathbf{v}(x)=\mathbf{a}+b\left(\begin{array}{c}
-x_{2} \\
x_{1}
\end{array}\right), \mathbf{a} \in \mathbb{R}^{2}, b \in \mathbb{R}\right\} & \text { if } d=2 .
\end{array}
$$

In this case we have the Korn inequality

$$
\|\mathbf{v}\|_{H^{1}} \leq C\|\mathbb{D} \mathbf{v}\|_{L^{2}} \quad \text { for all } \mathbf{v} \in H_{n}^{1}(\Omega),
$$

cf. Nečas [29, Theorem 3.5] for the case $d=3$. If $d=2$, the inequality follows from the three dimensional estimate by extending $\mathbf{v} \in H_{n}^{1}(\Omega)^{2}$ to $\tilde{\mathbf{v}}\left(x_{1}, x_{2}, x_{3}\right)=$ $\left(\mathbf{v}_{1}\left(x_{1}, x_{2}\right), \mathbf{v}_{2}\left(x_{1}, x_{2}\right), 0\right)^{T} \in H_{n}^{1}(\Omega \times(-1,1))$.

Because of

$$
\begin{aligned}
-(\operatorname{div}(2 \nu(c) \mathbb{D} \mathbf{v}), \mathbf{w})_{L^{2}(\Omega)} & =(2 \nu(c) \mathbb{D} \mathbf{v}, \mathbb{D} \mathbf{w})_{L^{2}(\Omega)}+(\gamma(c) \mathbf{v}, \mathbf{w})_{L^{2}(\partial \Omega)} \\
& =-\left(\mathbf{v}, \operatorname{div}(2 \nu(c) \mathbb{D} \mathbf{w})_{L^{2}(\Omega)}\right.
\end{aligned}
$$

for all $\mathbf{v}, \mathbf{w} \in \mathcal{D}\left(A_{\gamma}(c)\right), A_{\gamma}(c)$ is a symmetric operator. Moreover, if $\inf _{s \in \mathbb{R}} \gamma(s)=0$, then $\nu(s) \geq \nu_{0}>0$ and (A.3) implies

$$
-(\operatorname{div}(2 \nu(c) \mathbb{D} \mathbf{v}), \mathbf{v})_{L^{2}(\Omega)} \geq c_{0}\|\mathbf{v}\|_{H^{1}(\Omega)}^{2} \quad \text { for all } \mathbf{v} \in \mathcal{D}\left(A_{\gamma}\right)
$$

for some $c_{0}>0$. If $\gamma_{0}:=\inf _{s \in \mathbb{R}} \gamma(s)>0$, then one obtains

$$
-(\operatorname{div}(2 \nu(c) \mathbb{D} \mathbf{v}), \mathbf{v})_{L^{2}(\Omega)} \geq C\left(\gamma_{0}\right)\left(\|\mathbb{D} \mathbf{v}\|_{L^{2}(\Omega)}^{2}+\|\mathbf{v}\|_{L^{2}(\partial \Omega)}^{2}\right) \geq C^{\prime}\left(\gamma_{0}\right)\|\mathbf{v}\|_{H^{1}(\Omega)}
$$

because of $\|\mathbf{w}\|_{H^{1}(\Omega)} \leq C\|\mathbb{D} \mathbf{w}\|_{L^{2}(\Omega)}$ for any $\mathbf{w} \in H_{0}^{1}(\Omega)^{d}$. Furthermore, we have:

THEOREM A.1 Let $c \in W_{q}^{1}(\Omega), q>d$. Then $A_{\gamma}(c)$ is a positive self-adjoint operator on $L_{\sigma}^{2}(\Omega)$.

Proof: First of all, $A_{\gamma}(c): \mathcal{D}\left(A_{\gamma}\right) \rightarrow L_{\sigma}^{2}(\Omega)$ is invertible because of the following arguments: By the Lemma of Lax-Milgram for every $\mathbf{f} \in L_{\sigma}^{2}(\Omega)$ there is a unique $\mathbf{v} \in H_{n}^{1}(\Omega) \cap L_{\sigma}^{2}(\Omega)$ such that

$$
(2 \nu(c) \mathbb{D} \mathbf{v}, \mathbb{D} \mathbf{w})_{L^{2}(\Omega)}+(\gamma(c) \mathbf{v}, \mathbf{w})_{L^{2}(\partial \Omega)}=(\mathbf{f}, \mathbf{w})_{L^{2}(\Omega)} \quad \text { for all } \mathbf{w} \in \mathcal{D}\left(A_{\gamma}\right) .
$$

Since $\left.\gamma(c) \mathbf{v}\right|_{\partial \Omega} \in H^{\frac{1}{2}}(\partial \Omega)^{d}$, there is some $\mathbf{u} \in H^{2}(\Omega)^{d} \cap H_{0}^{1}(\Omega)^{d} \cap L_{\sigma}^{2}(\Omega)$ such that $(\mathbf{n} \cdot 2 \nu(c) \mathbb{D} \mathbf{u})_{\tau}=-\left.\gamma(c) \mathbf{v}\right|_{\partial \Omega}$ due to Lemma 2.3. By Gauß' theorem we obtain that $\tilde{\mathbf{v}}=\mathbf{v}+\mathbf{u}$ solves

$$
(2 \nu(c) \mathbb{D} \tilde{\mathbf{v}}, \mathbb{D} \mathbf{w})_{L^{2}(\Omega)}=(\mathbf{f}-\operatorname{div}(2 \nu \mathbb{D} \mathbf{u}), \mathbf{w})_{L^{2}(\Omega)} \quad \text { for all } \mathbf{w} \in \mathcal{D}\left(A_{\gamma}\right) .
$$


Because of [1, Theorem 5.2.3], we conclude that $\tilde{\mathbf{v}} \in H^{2}(\Omega)^{d}$, which yields $\mathbf{v} \in$ $H^{2}(\Omega)^{d}$. Since $A_{\gamma}(c)$ is symmetric due to (A.4), $A_{\gamma}(c)$ is self-adjoint.

Finally, we need:

Lemma A.2 Let $\gamma: \mathbb{R} \rightarrow[0, \infty)$ be continuously differentiable, $c \in W_{q}^{1}(\Omega)$ for some $q>d$, and assume that $\Omega$ possesses no axis of symmetry if $\inf _{s \in \mathbb{R}} \gamma(s)=0$. Then

$$
\left(L_{\sigma}^{2}(\Omega), \mathcal{D}\left(A_{\gamma}\right)\right)_{\frac{1}{2}, 2}=H^{1}(\Omega) \cap L_{\sigma}^{2}(\Omega) .
$$

Proof: We use that $A_{\gamma}(c)$ is an invertible, self-adjoint and positive operator on $L_{\sigma}^{2}(\Omega)$. Hence we can use Theorem 2.1 to conclude that for every $\mathbf{u}_{0} \in H^{1}(\Omega) \cap L_{\sigma}^{2}(\Omega)$ there is some $\mathbf{u} \in H^{1}\left(0, \infty ; L_{\sigma}^{2}(\Omega)\right) \cap L^{2}\left(0, \infty ; \mathcal{D}\left(A_{\gamma}\right)\right.$ such that $\left.\mathbf{u}\right|_{t=0}=\mathbf{u}_{0}$. Thus

$$
\left(L_{\sigma}^{2}(\Omega), \mathcal{D}\left(A_{\gamma}\right)\right)_{\frac{1}{2}, 2} \supseteq H^{1}(\Omega)^{d} \cap L_{\sigma}^{2}(\Omega)
$$

by the trace method of real interpolation. But the converse inclusion holds since for every $\mathbf{u} \in H^{1}\left(0, \infty ; L_{\sigma}^{2}(\Omega)\right) \cap L^{2}\left(0, \infty ; \mathcal{D}\left(A_{\gamma}\right)\right)$ we obviously have $\mathbf{u}_{0}=\left.\mathbf{u}\right|_{t=0} \in$ $H^{1}(\Omega)^{d} \cap L_{\sigma}^{2}(\Omega)$ because of $\left(L^{2}(\Omega), H^{2}(\Omega)\right)_{\frac{1}{2}, 2}=H^{1}(\Omega)$ and (2.4).

\section{References}

[1] H. Abels. Diffuse interface models for two-phase flows of viscous incompressible fluids. Lecture Notes, Max Planck Institute for Mathematics in the Sciences, No. 36/2007, 2007.

[2] H. Abels. Existence of weak solutions for a diffuse interface model for viscous, incompressible fluids with general densities. Comm. Math. Phys., 289(1):45-73, 2009.

[3] H. Abels. Longtime behavior of solutions of a Navier-Stokes/Cahn-Hilliard system. In Nonlocal and abstract parabolic equations and their applications, volume 86 of Banach Center Publ., pages 9-19. Polish Acad. Sci. Inst. Math., Warsaw, 2009.

[4] H. Abels. On a diffuse interface model for two-phase flows of viscous, incompressible fluids with matched densities. Arch. Rat. Mech. Anal., 194(2):463-506, 2009 .

[5] H. Abels. Nonstationary Stokes system with variable viscosity in bounded and unbounded domains. Discrete Contin. Dyn. Syst. Ser. S, 3(2):141-157, 2010.

[6] H. Abels and E. Feireisl. On a diffuse interface model for a two-phase flow of compressible viscous fluids. Indiana Univ. Math. J., 57(2):659-698, 2008. 
[7] H. Amann. Linear and Quasilinear Parabolic Problems, Volume 1: Abstract Linear Theory. Birkhäuser, Basel - Boston - Berlin, 1995.

[8] D. M. Anderson, G. B. McFadden, and A. A. Wheeler. Diffuse-interface methods in fluid mechanics. In Annual review of fluid mechanics, Vol. 30, volume 30 of Annu. Rev. Fluid Mech., pages 139-165. Annual Reviews, Palo Alto, CA, 1998.

[9] J. Bergh and J. Löfström. Interpolation Spaces. Springer, Berlin - Heidelberg New York, 1976.

[10] F. Boyer. Mathematical study of multi-phase flow under shear through order parameter formulation. Asymptot. Anal., 20(2):175-212, 1999.

[11] F. Boyer. Nonhomogeneous Cahn-Hilliard fluids. Ann. Inst. H. Poincaré Anal. Non Linéaire, 18(2):225-259, 2001.

[12] S. P. Chen and R. Triggiani. Proof of extensions of two conjectures on structural damping for elastic systems. Pacific J. Math., 136(1):15-55, 1989.

[13] L. de Simon. Un'applicazione della teoria degli integrali singolari allo studio delle equazioni differenziali lineari astratte del primo ordine. Rend. Sem. Mat. Univ. Padova, 34:205-223, 1964.

[14] C. G. Gal and M. Grasselli. Asymptotic behavior of a Cahn-Hilliard-NavierStokes system in 2D. Ann. Inst. H. Poincaré Anal. Non Linéaire, 27(1):401-436, 2010.

[15] C. G. Gal and M. Grasselli. Longtime behavior for a model of homogeneous incompressible two-phase flows. Discrete Contin. Dyn. Syst., 28(1):1-39, 2010.

[16] C. G. Gal and M. Grasselli. Trajectory attractors for binary fluid mixtures in 3d. Chin. Ann. Math. ser. B, 31:1-25, 2010.

[17] G. P. Galdi. An Introduction to the Mathematical Theory of the Navier-Stokes Equations, Volume 1. Springer, Berlin - Heidelberg - New York, 1994.

[18] M. Geißert, H. Heck, and M. Hieber. On the equation div $u=g$ and Bogovskiı's operator in Sobolev spaces of negative order. In Partial differential equations and functional analysis, volume 168 of Oper. Theory Adv. Appl., pages 113-121. Birkhäuser, Basel, 2006.

[19] M. E. Gurtin, D. Polignone, and J. Viñals. Two-phase binary fluids and immiscible fluids described by an order parameter. Math. Models Methods Appl. Sci., 6(6):815-831, 1996.

[20] B. Hanouzet. Applications bilinéaires compatibles avec un système à coefficients variables. Continuité dans les espaces de Besov. Comm. Partial Differential Equations, 10(4):433-465, 1985. 
[21] J. Johnsen. Pointwise multiplication of Besov and Triebel-Lizorkin spaces. Math. Nachr., 175:85-133, 1995.

[22] M. Kotschote. Strong solutions for a compressible fluid model of Korteweg type. Ann. Inst. H. Poincaré Anal. Non Linéaire, 25(4):679-696, 2008.

[23] S. G. Kreı̆n. Linear differential equations in Banach space. American Mathematical Society, Providence, R.I., 1971. Translated from the Russian by J. M. Danskin, Translations of Mathematical Monographs, Vol. 29.

[24] H.-G. Lee, J. S. Lowengrub, and J. Goodman. Modeling pinchoff and reconnection in a Hele-Shaw cell. I. The models and their calibration. Phys. Fluids, $14(2): 492-513,2002$.

[25] H.-G. Lee, J. S. Lowengrub, and J. Goodman. Modeling pinchoff and reconnection in a Hele-Shaw cell. II. Analysis and simulation in the nonlinear regime. Phys. Fluids, 14(2):514-545, 2002.

[26] C. Liu and J. Shen. A phase field model for the mixture of two incompressible fluids and its approximation by a Fourier-spectral method. Phys. D, 179(34):211-228, 2003.

[27] J. Lowengrub and L. Truskinovsky. Quasi-incompressible Cahn-Hilliard fluids and topological transitions. R. Soc. Lond. Proc. Ser. A Math. Phys. Eng. Sci., 454(1978):2617-2654, 1998.

[28] William McLean. Strongly elliptic systems and boundary integral equations. Cambridge University Press, Cambridge, 2000.

[29] J. Nečas and I. Hlavacek. Mathematical Theory of Elastic and Elastico-Plastic Bodies: An Introduction. Studies in Applied Mechanics 3, Elsevier Scientific Publishing Company, Amsterdam - Oxford - New York, 1981.

[30] A. Pazy. Semigroups of linear operators and applications to partial differential equations. Springer, Berlin-Heidelberg-New York, 1983.

[31] C. G. Simader and H. Sohr. A new approach to the Helmholtz decomposition and the Neumann problem in $L^{q}$-spaces for bounded and exterior domains. In Mathematical problems relating to the Navier-Stokes equation, volume 11 of Ser. Adv. Math. Appl. Sci., pages 1-35. World Sci. Publ., River Edge, NJ, 1992.

[32] H. Sohr. The Navier-Stokes equations. Birkhäuser Advanced Texts: Basler Lehrbücher. [Birkhäuser Advanced Texts: Basel Textbooks]. Birkhäuser Verlag, Basel, 2001. An elementary functional analytic approach.

[33] V. N. Starovoütov. On the motion of a two-component fluid in the presence of capillary forces. Mat. Zametki, 62(2):293-305, 1997. 
[34] E. M. Stein. Singular Integrals and Differentiability Properties of Functions. Princeton Hall Press, Princeton, New Jersey, 1970.

[35] H. Triebel. Interpolation Theory, Function Spaces, Differential Operators. North-Holland Publishing Company, Amsterdam, New York, Oxford, 1978.

[36] L. Zhao, H. Wu, and H. Huang. Convergence to equilibrium for a phase-field model for the mixture of two viscous incompressible fluids. Commun. Math. Sci., 7(4):939-962, 2009. 